\title{
Supramolecular organization in prokaryotic respiratory systems
}

Axel Magalon ${ }^{1,2}$, Rodrigo Arias-Cartin ${ }^{3}$ and Anne Walburger ${ }^{1,2}$

${ }^{1}$ CNRS, Laboratoire de Chimie Bactérienne (UMR7283), Institut de Microbiologie de la Méditerranée, CNRS, 13009 Marseille, France

${ }^{2}$ Aix-Marseille Univ, UMR7283, 13009 Marseille, France

${ }^{3}$ Department of Molecular, Cellular and Developmental Biology, Yale University, New Haven, CT 06520, USA

To whom correspondence should be addressed: Dr. Magalon Axel, Laboratoire de Chimie Bactérienne (UMR7283), Institut de Microbiologie de la Méditerranée, CNRS and AixMarseille Univ, 31 chemin Joseph Aiguier, 13009 Marseille, France, Tel.: (33) 491164668 ; Fax: (33) 491167194 ; E-mail: magalon@imm.cnrs.fr 
1. Introduction................................................................ 4

2. Supramolecular organization of the respiratory chains................... 6

3. Determinants for supramolecular organization.......................... 10

3.1. Lipids.................................................................. 10

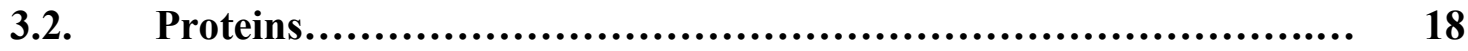

3.3. Subcellular localization................................................. 21

4. Implications of supramolecular organization............................ 24

5. Concluding remarks and future directions............................... 29

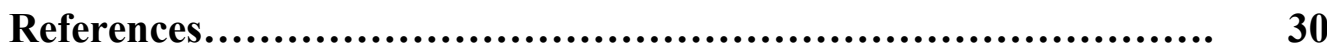




\begin{abstract}
:
Prokaryotes are characterized by an extreme flexibility of their respiratory systems allowing them to cope with various extreme environments. To date, supramolecular organization of respiratory systems appears as a conserved evolutionary feature as supercomplexes have been isolated in bacteria, archaea and eukaryotes. Most of the yet identified supercomplexes in prokaryotes are involved in aerobic respiration and share similarities with those reported in mitochondria. Supercomplexes likely reflect a snapshot of the cellular respiration in a given cell population. While the exact nature of the determinants for supramolecular organization in prokaryotes is not understood, lipids, proteins and subcellular localization can be seen as key players. Owing to the well-reported supramolecular organization of the mitochondrial respiratory chain in eukaryotes, several hypotheses have been formulated to explain the consequences of such arrangement and can be tested in the context of prokaryotes. Considering the inherent metabolic flexibility of a number of prokaryotes, cellular distribution and composition of the supramolecular assemblies should be studied in regards to environmental signals. This would pave the way to new concepts in cellular respiration.
\end{abstract}

\footnotetext{
ABBREVIATIONS

pmf: proton motive force, ADP: Adenosine diphosphate, ATP, Adenosine triphosphate, NADH: Nicotinamide adenosine dinucleotide, CL: Cardiolipin, PG : phosphatidylglycerol, PE : phosphatidylethanolamine, FBA : flux balance analysis, ROS : Reactive oxygen species, ACIII : Alternative complex III, HIPIP : High-potential iron-sulfur protein
} 


\section{Introduction}

Respiration is a catabolic process that is fundamental to all kingdoms of life. Schematically, electrons are extracted from respiratory substrates and transferred through a succession of multimeric protein complexes. This electron transfer is coupled with the translocation of protons across the membrane and thus to the generation of a transmembrane electrochemical proton gradient or proton motive force $(\mathrm{pmf})$ that drives the phosphorylation of ADP into ATP. In the mitochondrial respiratory system, electrons migrate from NADH or succinate to oxygen through four complexes (I to IV) localized in the inner mitochondrial membrane $<$ Figure 1>. Complex I and complex II transfer electrons to quinones from NADH or succinate, respectively. In addition complex I contributes to the maintenance of the electrochemical gradient by pumping protons across the membrane during quinone reduction. The reduced quinol pool constitutes an electron and proton reservoir that can be used by the cytochrome $b c_{1}$ complex (complex III). Based on a Q-cycle functioning, this complex couples quinol oxidation to cytochrome $c$ reduction with the concomitant net translocation of protons across the membrane. The reduced mobile cytochrome $c$ transfers electrons to cytochrome $c$ oxidase (complex IV) which couples proton translocation to oxygen reduction. Overall, the transfer of 2 electrons issued from NADH to oxygen results in 10 protons being translocated across the membrane.

The structural and functional organization of the mitochondrial respiratory chain has been a matter of much debate for several decades. As such, several models have been proposed ranging from a "liquid state" model or "random collision" model which predicts that all complexes would be randomly dispersed in the lipid bilayer and would be functionally connected by mobile carriers such as quinone and cytochrome $c$ (reviewed in (Hackenbrock et al., 1986; Bianchi et al., 2004; Lenaz and Genova, 2009) (Figure 1A) to a "solid state" model (Chance and Williams, 1955) where the respiratory complexes are organized in bigger structures within the inner mitochondrial membrane (Figure 1B). While the "liquid state" model was supported by the ability to isolate functionally active individual complexes, the observation that complexes can stably interact within entities named supercomplexes is in favor of the "solid state" one. The existence of supercomplexes was supported by the emergence of mild isolation techniques of membrane components (Schagger and Pfeiffer, 2000). Many supercomplexes from a wide variety of organisms and organs have since been biochemically characterized and differ by a large variety of stoichiometries and complex compositions. Of most interest, Acin-Perez et al isolated a functional "respirasome" from 
mammal cells ensuring autonomously electron transfer from NADH or succinate to oxygen and containing all four complexes together with quinones and cytochrome $c$ (Acin-Perez et al., 2008). To date, the emerging picture is between both of these extreme situations depicted in each model as they likely coexist within mitochondria with reported variation in the abundance and composition of supercomplexes in response to physiological changes ((Gomez et al., 2009; Helbig et al., 2009; Rosca and Hoppel, 2009; Frenzel et al., 2010; Gomez and Hagen, 2012). Taken together these information lead to the idea that when supercomplexes are identified, they reflect a snapshot of the cellular respiration in a given cell population.

A higher level of supramolecular organization was recently demonstrated in the inner mitochondrial membrane with the spatial segregation of the ATP synthase and of the respiratory complexes (Dudkina et al., 2010b; Davies et al., 2011). Long rows of ATP synthase dimers were found exclusively on curved cristae edges in accordance with the reported influence of oligomerization of this complex on the membrane curvature (Paumard et al., 2002; Dudkina et al., 2005; Minauro-Sanmiguel et al., 2005; Dudkina et al., 2006b; Strauss et al., 2008). Interestingly, complex I appeared in distinct regions of the cristae membrane, ie the flat cristae membrane regions. Moreover, such arrangement conserved in bovine heart, potato and fungi may support functional implications (Davies et al., 2011). These data fueled a third model of respiratory system organization, the "compartment model" (Figure 1C). In this model, the ATP synthase participates directly to the morphogenesis of the inner mitochondrial membrane which in turn could influence the whole functioning of the mitochondrial respiratory chain. Indeed, such an organization would favor higher concentrations of protons, cytochrome $c$ or other cofactors as well as collisions between electron donors and acceptors.

While the understanding of the physical organization of the respiratory mitochondrial chain is still under progress, it is now well accepted that supercomplexes exist and can function as respirasomes. Supercomplex formation is necessary for assembly and stability of its individual components (Acin-Perez et al., 2004; Schagger et al., 2004; D'Aurelio et al., 2006) and may influence inner membrane morphogenesis (Hackenbrock, 1968; Vonck and Schafer, 2009). However, the functional advantages resulting from such organization remain much debated. It was proposed that supercomplexes allow substrate channeling between associated complexes, confinement of the mobile carriers and limiting reactive oxygen species production as a byproduct of oxidative phosphorylation (Lenaz and Genova, 2010) even though direct evidences are still lacking. Recently, Trouillard et al showed that cytochrome $c$ is not trapped within supercomplexes and encounters no particular restriction to its diffusion 
and that destabilization of either the mitochondrial cristae structure or the supercomplexes did not result in perturbation of the electron transfer kinetics (Trouillard et al., 2011). Overall, the functional relevance of supramolecular assemblies remains an open question.

In prokaryotic cells, respiratory enzymes react as mitochondrial respiratory complexes as they allow formation of a proton gradient used for ATP synthesis. However, as prokaryotes are able to colonize multiple environments, prokaryotic respiratory enzymes are very diverse in term of structure and reactivity (Simon et al., 2008). Besides oxygen, they can use multiple alternative electron acceptors. Another marked difference is the coexistence of several enzymes both at the electron input and output leading to a branched character of their respiratory chains $<$ Figure $2>$. Such promiscuity of the electron transfer between different complexes may be taken as an advantage for increasing the metabolic flexibility and ability to cope with rapidly changing environments. On the opposite, confinement obtained through the existence of supercomplexes may also be beneficial. Nevertheless, supramolecular organization of prokaryotic respiratory chains has been underestimated for decades. This is not specific to respiratory complexes as prokaryotes have long been thought to be noncompartmentalized. With the recent improvements in fluorescence live cell imaging, it has been demonstrated that prokaryotes are highly organized and that most of their molecules including proteins, lipids and nucleic acids are present in cellular subdomains at which they perform their function (Rudner and Losick, 2010; Nevo-Dinur et al., 2012). In this context, this review aims to provide an actual view on supramolecular organization of prokaryotic respiratory systems, their determinants and to discuss the functional and cellular implications of it.

\section{Supramolecular organization of the respiratory systems}

In regards to the exceptional diversity in membrane-bound complexes participating to respiration in archaea or bacteria, only few examples of supramolecular organization have been reported. Examples are found both in archaea and bacteria yet covering only a part of their respective existing phyla $<$ Table $1>$. Hence, amongst archaea, four species belonging to the crenarchaeota (Sulfolobus sp. strain 7, Acidianus ambivalens, Pyrodictium abyssi and Thermoproteus neutrophilus) were reported to contain supercomplexes (Iwasaki et al., 1995; Dirmeier et al., 1998; Laska and Kletzin, 2000; Laska et al., 2003). In bacteria, examples were reported in gram positive organisms such as actinobacteria (Corynebacterium glutanicum and Mycobacterium smegmatis) (Niebisch and Bott, 2003; Megehee et al., 2006) and firmicutes 
(bacterium PS3) (Sone et al., 1987; Tanaka et al., 1996) as well as in gram negative ones such as aquificae (Aquifex aeolicus) (Guiral et al., 2005; Guiral et al., 2009; Prunetti et al., 2010; Gao et al., 2012), bacteroidetes (Rhodothermus marinus) (Refojo et al., 2010a; Refojo et al., 2010b), $\alpha$-proteobacteria (Paracoccus denitrificans and Bradyrhizobium japonicum) (Berry and Trumpower, 1985; Keefe and Maier, 1993; Stroh et al., 2004) and $\gamma$-proteobacteria (Escherichia coli and Acidithiobacillus ferrooxidans) (Castelle et al., 2008; Lenn et al., 2008b; Sousa et al., 2011). At this stage, one could consider that a supramolecular organization of the respiratory complexes in both archaea and bacteria is an evolutionary conserved trait for which selective advantages remain to be established. However, prokaryotic respiratory chains are dynamic entities that display great variability in their components. How to reconcile such apparent contradiction ie a great variability with the reported existence of a supramolecular organization?

Interestingly, most of the reported supercomplexes of the aerobic respiratory chain in prokaryotes include the highly conserved complexes III and IV (Berry and Trumpower, 1985; Sone et al., 1987; Keefe and Maier, 1993; Iwasaki et al., 1995; Niebisch and Bott, 2003; Guiral et al., 2009; Gao et al., 2012) (Table 1). In fact, cytochrome $b c_{1}$ (complex III) is one of the mostly represented membrane-bound complexes in supercomplexes. This enzyme complex is constituted by three redox-active subunits: a cytochrome $b$ with two $b$-type hemes, the Rieske protein with a FeS cluster and cytochrome $c_{1}$ with a covalently bound heme $c$ (Berry et al., 2000). A functional association of the cytochrome $b c_{1}$ complex with a $a a_{3}$-type cytochrome $c$ oxidase giving rise to oxygen uptake upon quinol oxidase activity was repeatedly reported in several bacteria, i.e. Paracoccus denitrificans (Berry and Trumpower, 1985), the thermophilic bacterium PS3 (Sone et al., 1987) or in the thermoacidophilic archaeon Sulfolobus sp. Strain 7 (Iwasaki et al., 1995). In some instances, variations of the theme have been described with the formation of a $b c c-a a_{3}$ supercomplex with quinol-driven $\mathrm{O}_{2}$ reduction in Mycobacterium smegmatis (Megehee et al., 2006) and Corynebacterium glutanicum (Niebisch and Bott, 2003) where the $c_{1}$ subunit of the classical $b c_{1}$ complex harbors two cytochromes $c$ instead of one. The immediate functional consequence is that this respiratory chain does not required a separate mobile cytochrome $c$ for electron transfer between the cytochrome $c_{1}$ to the $\mathrm{Cu}_{\mathrm{A}}$ center in the cytochrome $a a_{3}$. Supramolecular organization is, in this context, beneficial as electron transfer requires intimate contact between complexes (Bott and Niebisch, 2003). Another example is the isolation of a supercomplex in the highly thermophilic bacterium Aquifex aeolicus between a cytochrome $b c_{1}$ complex and a cytochrome $b a_{3}$-type oxidase (Guiral et al., 2009), the latter displaying the 
unique property to oxidize both reduced cytochrome $c$ and ubiquinol (Gao et al., 2012). To date, such dual substrate specificity appears not to be a general property of heme-copper terminal oxidases but to be restricted to A. aeolicus. Finally, in Bradyrhizobium japonicum, a functional association between the cytochrome $b c_{1}$ complex and a $c b b_{3}$-type cytochrome $c$ oxidase was reported (Keefe and Maier, 1993). Overall, prokaryotes have developed many alternatives to shuttle electrons between complexes III and IV with the participation of soluble or membrane-bound cytochromes $c$ or by precluding any electron shuttle with the fusion of an additional heme $c$ domain to one constituent of the two complexes (Niebisch and Bott, 2003; Janzon et al., 2007) or by using high-potential iron-sulfur protein (HIPIP) (Pereira et al., 1999b; Pereira et al., 1999a). While supercomplex organization has not been systematically reported in the situation where no electron shuttle is present, it can be tentatively considered that this is likely to occur for optimal electron transfer between both complexes. The situation described in $R$. marinus belonging to the bacteroidetes phylum provides a good illustration of this idea. Interestingly, this organism is characterized by the existence of an alternative complex III (ACIII) catalyzing essentially the same reaction ie coupling quinol oxidation with cytochrome $c$ reduction but not belonging to the same family. Moreover, this complex appears to be widespread in the bacteria domain (Refojo et al., 2010a). Refojo et al reported the functional association of the ACIII with the $\mathrm{Caa}_{3}$-type oxygen reductase in $R$. marinus (Refojo et al., 2010b). A monoheme subunit of the ACIII complex is proposed to serve as electron donor to the oxidase and to perform an equivalent role to cytochrome $c$. A supramolecular organization of complexes III and IV was also shown in mitochondria from yeast (Schagger and Pfeiffer, 2000), plants (Eubel et al., 2004a; Krause et al., 2004; Dudkina et al., 2006a) and mammals (Schagger and Pfeiffer, 2000; Stuart, 2008; Chen et al., 2012). Hence, quinol:oxygen oxidoreductase supercomplexes were detected in all branches of the tree of life supporting the idea that this highly organized state is a general feature of living organisms. Apart from the quinol:oxygen oxidoreductase supercomplex, other functional associations of components of aerobic respiratory chains have been reported (Table 1). In P. denitrificans, an additional supercomplex made up of the NADH dehydrogenase, the cytochrome $b c_{1}$ complex and the $a a_{3}$-type cytochrome $c$ oxidase was reported (Stroh et al., 2004). The membranebound cytochrome $c_{552}$ most likely mediating electron transfer between the $b c_{1}$ and $a a_{3}$-type cytochromes is also part of the supercomplex while not being essential for its formation. The supercomplex appears to contain approximately one complex I, four copies of the complexes III and IV while the cytochrome $c_{552}$ was substoichiometric. Such supramolecular organization recalls the one reported in mitochondria which couples $\mathrm{NADH}$ oxidation to $\mathrm{O}_{2}$ 
reduction (Schagger and Pfeiffer, 2000). Interestingly this supercomplex does not include other components of the aerobic respiratory chain of $P$. denitrificans such as the $c b b_{3}$-type cytochrome $c$ oxidase.

In Escherichia coli, several supercomplexes were identified: one made up of the NADH dehydrogenases NDH-1 and NDH-2, and another composed by two quinol:oxygen reductases, cytochrome $b_{3}$ and $b d$-I, together with the formate dehydrogenase O (FdoGHI) (Sousa et al., 2011). Nevertheless, the functional association of these supercomplexes was not assessed through activity measurements and precludes any functional interpretation. Indeed while the physical association of FdoGHI, a quinone reductase, with two distinct quinol oxidases may result in the formation of a respiratory chain coupling formate oxidation with oxygen consumption, the supercomplex made up of the two alternatives NADH dehydrogenases seems to date meaningless and calls for additional functional studies. Similarly, Guiral et al (Guiral et al., 2009) reported the isolation of several supercomplexes in the highly thermophilic bacterium $A$. aeolicus. In addition to the functional association between the cytochrome $b c_{1}$ complex and the cytochrome $b a_{3}$-type oxidase, putative associations of complexes involved in oxygen reduction such as sulfide quinone reductase, cytochrome $b c_{1}$ complex, cytochrome $c$ oxidase, quinol oxidase and membrane-bound hydrogenase were suspected due to their presence within the same gel band after blue native PAGE of solubilized membranes (Guiral et al., 2009). These complexes would reflect two distinct electron transfer pathways allowing oxygen reduction with either $\mathrm{H}_{2} \mathrm{~S}$ or $\mathrm{H}_{2}$ as electron donors. Further support to this model came from the isolation of a multienzyme supercomplex supporting oxygen reduction in the presence of the electron donor $\mathrm{H}_{2} \mathrm{~S}$ (Prunetti et al., 2010). The supercomplex isolated from A. aeolicus cells grown in $\mathrm{H}_{2} / \mathrm{S}^{\circ} / \mathrm{O}_{2}$ medium is constituted by a dimeric $b c_{1}$ complex, a $b a_{3}$-type cytochrome $c$ oxidase, one or two sulfide quinone reductases, traces of the monoheme cytochrome $c_{555}$ and quinones. More interesting is the isolation of a supercomplex spanning the outer and inner membranes of the acidophilic bacterium Acidithiobacillus ferrooxidans that had iron oxidase as well as oxygen reductase activities (Castelle et al., 2008). This supercomplex allowing electron transfer from Fe(II) to $\mathrm{O}_{2}$ is notably constituted by the outer membrane-bound cytochrome $c \mathrm{Cyc}_{2}$, the inner membrane-bound cytochromes $\mathrm{Cyc}_{1}$ and $\mathrm{Cyc}_{42}$ as well as the $b c_{1}$ complex and the cytochrome $c$ oxidase. Such an organization may allow electron transfer "downhill" towards $\mathrm{O}_{2}$ involving the cytochrome $c$ oxidase or "uphill" to NADH via the cytochrome $b c_{1}$ complex, respectively (Bird et al., 2011). Finally, a physical interaction has been reported in reconstituted systems between the $\mathrm{F}_{1} \mathrm{~F}_{0}$-ATP synthase and the $\mathrm{caa}_{3}$ oxygen reductase in the alkaliphilic Bacillus 
pseudofirmus OF4 which supports the idea of a sequestered path of protons in such organisms for energy conservation (Liu et al., 2007).

Noteworthy is the fact that only few studies have reported the isolation and characterization of supercomplexes involved in electron transfer using alternative electron acceptors to molecular oxygen (Table 1). A functional association allowing reduction of $\mathrm{S}^{\circ}$ with $\mathrm{H}_{2}$ to $\mathrm{H}_{2} \mathrm{~S}$, including a hydrogenase and a sulfur reductase was reported in A. aeolicus (Guiral et al., 2005) and in

three hyperthermophilic archaeons, Pyrodictium abyssi (Dirmeier et al., 1998), Thermoproteus neutrophilus (Laska and Kletzin, 2000) and the acidophilic Acidianus ambivalens (Laska et al., 2003).

In summary of this section, the supramolecular organization of components of the respiratory process has been reported in a yet restricted number of prokaryotes. Information is essentially obtained through purification of complexes under mild detergent conditions allowing the isolation of supercomplexes and their subsequent analysis by blue native PAGE, UV-vis spectroscopy and enzymatic activity measurements. In some cases, the stoichiometry can be estimated but a detailed structural analysis of the isolated and studied supercomplexes is so far lacking. To our knowledge, a single study has reported intra- and inter-complex interactions between subunits of the $b c_{1}-a a_{3}$ supercomplex isolated from the thermophilic Bacillus PS3 through the use of crosslinkers (Tanaka et al., 1996). Moreover, information about the nature and amount of lipids and quinones associated within the isolated supercomplexes is lacking and needs to be further considered in the future as it can provide critical information in regards to the functionality of such assemblies.

\section{Determinants for supramolecular organization}

\subsection{Lipids}

In addition to providing specialized permeability barriers, biological membranes should also be seen as a dynamic non-covalent supramolecular organization of individual lipid molecules whose combined physical and chemical properties define the matrix within which membrane proteins are organized. Besides, lipid environment is very critical in fixing the biophysical and biochemical properties of numerous intrinsic proteins. It is widely accepted that specific protein-lipid interactions are very important for the structural and functional integrity of many eukaryotic and prokaryotic membrane proteins. If the bulk of lipid molecules in biological membranes acts as solvent for integral membrane proteins and as such does not display any 
specific interaction with them, others form an annulus or even are specifically integrated between and within the subunits of oligomeric protein complexes (Ernst et al., 2010). In particular, phospholipids are structurally and functionally important components in the energy-transducing multimeric complexes. Furthermore biological membranes are crowded (Ryan et al., 1988; Engelman, 2005) and transmembrane proteins might thus be able to interfere with themselves through the surrounding lipid environment (Domanski et al., 2012). In this context, cardiolipin (CL) is seen at present as a key component of energy-transducing membranes for several reasons. At first, this peculiar lipid is found in the mitochondrial inner membrane, the chloroplast, the hydrogenosomes or the cytoplasmic membrane of prokaryotes (Depalo et al., 2004; de Andrade Rosa et al., 2006; Ventrella et al., 2007; Corcelli, 2009; Mileykovskaya and Dowhan, 2009). CL has also the ability to interact with a wide variety of membrane proteins in eukaryotes, most of them being related to oxidative phosphorylation or photophosphorylation, thereby modulating their catalytic activities and/or providing stability (Fry and Green, 1980; Yu and Yu, 1980; Poore and Ragan, 1982; Eble et al., 1990; Robinson et al., 1990; Schagger et al., 1990; Hayer-Hartl et al., 1992; Robinson, 1993; Beyer and Nuscher, 1996; Gomez and Robinson, 1999; Sedlak and Robinson, 1999; Depalo et al., 2004; Ventrella et al., 2007). In prokaryotes, CL has also been shown to restore the activity of a number of purified respiratory complexes such as the NADH dehydrogenase (Dancey and Shapiro, 1977; Esfahani et al., 1977; Thomson and Shapiro, 1981), the lactate dehydrogenase (Tanaka et al., 1976; Esfahani et al., 1977; Kimura and Futai, 1978), the succinate dehydrogenase (Esfahani et al., 1977; Reddy and Weber, 1986), the cytochrome $b o_{3}$ ubiquinol oxidase (Kita et al., 1984) or the nitrate reductase A (Arias-Cartin et al., 2011). Not surprisingly, several X-ray structures have reported the presence of a tightly bound CL molecule to the formate dehydrogenase $\mathrm{N}$ (Jormakka et al., 2002b) and succinate dehydrogenase (Yankovskaya et al., 2003; Horsefield et al., 2006) in E. coli. More recently, a $\mathrm{CL}$ molecule has been considered to be present in the X-ray structure of the E. coli nitrate reductase A (Arias-Cartin et al., 2011).

Furthermore, it has been proposed that CL can play an important role in the oligomerization of these energy-transducing complexes. This is typically the case of the trimeric assembly of the E. coli formate dehydrogenase N (FdnGHI) (Jormakka et al., 2002b) where CL is found not only at the interface of the trimer but also between subunits within each monomer. The acyl chains of CL are at the crossroad of all this architecture. While one of the acyl chains fills in a tunnel leading to the heme $b_{\mathrm{c}}$ in proximity of the quinone-binding site, others are involved in interactions with neighboring subunits. In this context, CL seems essential for 
both trimer formation and inter-subunits interactions. The proximity of one acyl chain with the heme in charge of quinone reduction allows to postulate that $\mathrm{CL}$ is also required for enzyme functioning (Arias-Cartin et al., 2012).

A similar situation is likely encountered in the case of the E. coli nitrate reductase A complex (NarGHI) (Bertero et al., 2003). Indeed, the crystal packing of NarGHI revealed a dimer with a two-fold symmetry axis approximately parallel to the membrane normal. A phospholipid molecule yet undefined is present at the interface of the dimer, and NarI from one heterotrimer together with the swapped domain of NarH from the other heterotrimer stabilize this lipid. Using a large excess of detergent was not sufficient to resolve the dimeric structure of the NarGHI complex nor the release of a CL molecule found in stoichiometric amount in the nearly inactive detergent-solubilized complex (Arias-Cartin et al., 2011). The nature of the lipid present at the dimer interface can thus be tentatively attributed to a CL molecule. This CL molecule would thus play a role in the dimerization of the NarGHI heterotrimer in the same way as in complexes III, IV and V (Eble et al., 1990; Lange et al., 2001; Palsdottir et al., 2003; Qin et al., 2006; Shinzawa-Itoh et al., 2007) or the ADP/ATP carrier (Nury et al., 2005). For instance, the dimeric state of the bovine complex IV is strongly stabilized by CL making extensive contacts with each monomer (Shinzawa-Itoh et al., 2007). It is worth mentioning that binding of a second CL molecule to a distinct cavity within the NarGHI complex is responsible for enzyme functionality (Arias-Cartin et al., 2011).

In contrast, the situation appears to be different in the case of the E. coli succinate dehydrogenase complex. The X-ray crystal structure of the E. coli succinate dehydrogenase (SdhABCD) was solved at a resolution of $2.6 \AA$ and shows a trimeric structure (Yankovskaya et al., 2003). In particular, a CL molecule is literally engulfed in the SdhC and SdhD interface and occupy the hydrophobic space above the heme $b_{\mathrm{P}}$ which accommodates an additional heme $b$ in $W$. succinogenes quinol:fumarate oxidoreductase structure (Lancaster et al., 1999). The CL phosphatidyl groups point toward the periplasmic side of the membrane and are maintained by direct hydrogen bonds with residues originating from both SdhC and SdhD transmembrane subunits. As compared to the E. coli FdnGHI heterotrimer, the role of CL in the trimer formation of SdhABCD remains unclear owing to its particular positioning and the absence of contact of the acyl chains with neighboring monomers. Recently, Ruprecht and coworkers reported additional X-ray structures of the E. coli SdhABCD complex which do not show convincing electron densities for lipid molecules in the region where CL was first identified (Ruprecht et al., 2009; Ruprecht et al., 2011). As expected, the absence of CL leaves behind a considerable hydrophobic groove in these structures and an increased $B$-factor 
of the transmembrane subunits SdhCD. From these observations the authors claimed a stabilizing role of CL in these membrane-embedded proteins while being not essential for integrity of heme $b$. Recently, the mitochondrial complex II from the yeast Saccharomyces cerevisiae was shown to require CL for optimal function and assembly (Schwall et al., 2012). In particular, $\mathrm{CL}$ is required to maintain the interaction between the catalytic dimer and membrane subunits, and to promote electron transfer from succinate oxidative site to the quinone binding site. In light of these results, the function of CL in E. coli SdhABCD should be reconsidered since earlier studies have reported the beneficial effect of CL towards succinate dehydrogenase activity of the isolated complex (Esfahani et al., 1977).

A number of energy-transducing complexes of prokaryotic respiratory chains have been reported to exist in oligomeric assemblies but, to date information concerning the influence of lipids and of specific ones on such supramolecular organization is scarce (see for instance, (Jormakka et al., 2002b; Esser et al., 2008; Jormakka et al., 2008; Kleinschroth et al., 2011). Nonetheless, as exemplified above, it is likely that specific lipids such as CL, to a certain extent, influence the oligomerization of individual complexes. Conversely, protein-induced perturbations of the membrane shape such as influencing bilayer thickness or bending will lead to attraction of proteins in some instances. The consequence of such lipid-protein interactions is their ability to induce cooperativity in the conformational changes of the neighbouring membrane proteins and in their oligomerization (Phillips et al., 2009). One of the most prominent examples is the oligomeric state of ATP synthase which plays a role in cristae formation and morphology in mitochondria (Paumard et al., 2002; Soubannier et al., 2002). It is widely accepted that dimerization of ATP synthase induces bending of the inner mitochondrial membrane and that its oligomerization further constraints the membrane curvature. Recently, Acehan et al (Acehan et al., 2011a) demonstrated that the lipid composition of the mitochondrial inner membrane, specifically the presence of CL, dramatically influences ATP synthase oligomerization. Such a situation may even extend to peripheral membrane proteins, a number of which being components of respiratory chains, shown to transiently form highly ordered structures due to membrane-mediated interactions (Morozova et al., 2011). Hence, oligomerization of prokaryotic respiratory complexes resulting or not on CL binding may also result in local perturbations of the membrane shape and in its lipid composition.

Overall, after having reported that CL may contribute to the oligomerization of several prokaryotic energy-transducing complexes, reorganization of these structures may also be at the origin of the formation of supercomplex entities. Recently, high-resolution cryo-electron 
microscopy reconstructions of the bovine $\mathrm{I}_{1} \mathrm{III}_{2} \mathrm{IV}_{1}$ supercomplex were completed allowing an unprecedented analysis of the respirasome carrying out electron transfer from NADH to molecular oxygen (Althoff et al., 2011; Dudkina et al., 2011). In particular, repositioning of the complex IV monomer has been made with the contact interface involved in the dimer facing outwards the supercomplex. More interesting is the fact that a gap is clearly visible in the membrane region between individual complexes, in particular III and IV with almost no protein-protein interactions. The lower map density obtained in the membrane parts of the three component complexes can be attributed to a high lipid content of the purified supercomplex while it cannot be ruled out the presence of additional small size proteins. Hence, specific lipid contacts are likely to play a major role in stabilization of the supercomplex. For instance, CL is considered to play an active role in supramolecular organization of the mitochondrial respiratory chain through stabilization of supercomplexes (Zhang et al., 2002; Pfeiffer et al., 2003; Eubel et al., 2004b; Mileykovskaya et al., 2005; Zhang et al., 2005; McKenzie et al., 2006; Xu et al., 2006; Stuart, 2008; Vonck and Schafer, 2009; Dudkina et al., 2010a) or in the oligomerization of the ATP synthase responsible for the cristae morphology (Acehan et al., 2011a). In this context, CL binding to a number of anaerobic respiratory complexes in bacteria, such as formate dehydrogenase $\mathrm{N}$ (FdnGHI) (Jormakka et al., 2002b) and nitrate reductase A (NarGHI) (Arias-Cartin et al., 2011) may have additional roles. These two complexes constitute a major alternative respiratory pathway in several prokaryotes, the nitrate respiratory pathway, induced by anaerobiosis and the presence of nitrate (Berg and Stewart, 1990). Reorganization of the oligomeric structures of both FdnGHI and NarGHI might allow formation of a supercomplex where the contact interface of FdnGHI involved in CL binding within the heterotrimer interacts with the NarGHI complex. Such organization may position the quinone binding site of FdnGHI (as seen from HQNO binding, a menaquinol analog inhibitor) in proximity to the quinol-binding site cavity $Q_{D}$ within NarGHI. Such arrangement resulting in short diffusion distances for quinone molecules may increase the electron transfer efficiency from FdnGHI to NarGHI as proposed in the solid-state model. Finally, the reported co-purification of FdnGHI and NarGHI complexes over a wide-range of conditions (Enoch and Lester, 1975; Jormakka et al., 2002a) may be suggestive of their physical association. Furthermore, Claypool et al (Claypool et al., 2008; Claypool, 2009) reported that CL defines the functional interactome of the mitochondrial ADP/ATP carrier known to associate with the respiratory supercomplexes. Overall, the participation of anionic phospholipids and in particular CL to functioning, 
defining the interactome or supramolecular organization of the bacterial respiratory chains remains largely unexplored.

An additional level of complexity is attained when considering that most biological membranes contain a mixture of lipid molecules differing in their polar headgroups and in their fatty acyl chains. Furthermore, the lipid composition of the bacterial membrane varies considerably with growth conditions. Although $\mathrm{CL}$ is a minor component during the exponential bacterial growth phase, its level increase in the stationary phase (Cronan, 1968; Randle et al., 1969; Short and White, 1971; Shibuya, 1992; Hiraoka et al., 1993; Lopez et al., 2006; Koprivnjak et al., 2011), in response to energy deprivation (Koch et al., 1984), osmotic stress (Kanemasa et al., 1972; McGarrity and Armstrong, 1975; Miller, 1986; Lopez et al., 1998; Catucci et al., 2004; Lopez et al., 2006; Romantsov et al., 2009) or external pH (Card and Trautman, 1990; Macgilvray et al., 2012). Therefore changing the lipid composition would dramatically change the oligomerization status of energy-transducing complexes which exhibit preference for a given lipid species. In support of this idea, absence of CL leads to a severe reduction of supercomplexes assembly and stability in mitochondria (Pfeiffer et al., 2003; Zhang et al., 2005). By the same token, alterations of CL structure, content and acyl chain composition during aging, apoptosis, cancer or Barth syndrome are also associated with a defective mitochondrial respiratory chain and a dramatic change in its supramolecular organization (McMillin and Dowhan, 2002; Petrosillo et al., 2003a; Petrosillo et al., 2003b; Paradies et al., 2004; McKenzie et al., 2006; Schlame and Ren, 2006; Kiebish et al., 2008; Petrosillo et al., 2008; Petrosillo et al., 2009; Schlame and Ren, 2009; Paradies et al., 2011).

In contrast, the question of the relations between lipid composition of the cytoplasmic membrane and functioning or supramolecular organization of the bacterial respiratory chains has never been directly addressed. Apart from the relatively limited level of information on supercomplexes in prokaryotes as compared with eukaryotes, one of the reasons can be found by the fact that both anionic lipids, PG and CL are dispensable in E. coli used as a model organism owing to its simple lipid composition (Miyazaki et al., 1985; Matsumoto, 2001). Indeed, $E$.coli membranes contain three major phospholipids: phosphatidylethanolamine (PE), a zwitterionic lipid which represents up to $70 \%$ of the total lipid content as well as two anionic ones, phosphatidylglycerol (PG) (20-25\%) and CL (5-10\%). In particular, lack of PG and $\mathrm{CL}$ is associated with an increase of the doubling times under aerobic conditions on both rich and minimal media (Asai et al., 1989; Kikuchi et al., 2000) as well as with a reduction of the cell size by nearly $20 \%(2.93 \mu \mathrm{m}$ vs $3.66 \mu \mathrm{m}$ for the parental strain) (Mileykovskaya et al., 2009). Interestingly, a similar observation was made in Pseudomonas putida with a strong 
reduction of the cell size in the cls mutant strain $(1.9 \mu \mathrm{m}$ vs $2.8 \mu \mathrm{m}$ for the wild-type strain) (Bernal et al., 2007). Null mutants of the $c l s$ gene encoding for the major cardiolipin synthase in $E$. coli were found to only have a negligible growth defect despite an almost total lack of CL (Shibuya et al., 1985; Nishijima et al., 1988). One explanation for the traces of CL in the cls mutant may be due to the existence of a second protein with CL synthase activity, YbhO but it remains unclear whether it contributes in vivo to CL cell content (Guo and Tropp, 2000). While the E. coli mutant strain lacking both PG and CL is viable under aerobic conditions probably due to the presence of two other anionic phospholipids (Mileykovskaya et al., 2009), it remains open whether the combined defect in PG and CL has an influence on anaerobic growth and on the functionality of respiratory complexes. In this context, the nitrate reductase A (NarGHI), a cytoplasmic membrane-bound quinol-nitrate oxidoreductase that terminates the respiratory chain developed by $E$. coli when grown anaerobically with nitrate as terminal electron acceptor, strictly requires CL binding for functionality (Arias-Cartin et al., 2011). Mechanistically, it is considered that CL binding tunes the interaction with the quinol substrate by promoting a correct positioning of heme $b_{\mathrm{D}}$ and of its ligand His66 involved in the stabilization of the semiquinone intermediates (Arias-Cartin et al., 2011).

As compared with the anionic phospholipids, elimination of PE via a pssA mutant shows a severe growth defect associated with filamentation of the E. coli cells (DeChavigny et al., 1991). However, supplementation of the growth medium with a subset of divalent cations in mmolar amounts $\left(\mathrm{Ca}^{2+}, \mathrm{Mg}^{2+}\right.$ or $\left.\mathrm{Sr}^{2+}\right)$ restores growth while the cells are still filamentous. It is considered that the lack of PE affects the localization and correct assembly of the cell division apparatus (Mileykovskaya et al., 1998). Additional consequences of the absence of PE are a defective topology of a subset of integral membrane proteins among which the lactose permease LacY is the most prominent example (Bogdanov et al., 2002; Dowhan and Bogdanov, 2009). Upon growth on rich media supplemented with $\mathrm{MgCl}_{2}$ under aerobic conditions, unaffected $\mathrm{pH}$ gradients driven by succinate, lactate or ATP were reported using inverted membrane vesicles (Mileykovskaya and Dowhan, 1993). As such, the structural integrity of the corresponding electron transfer chains and of the cytoplasmic membrane is preserved. On the contrary, the cytochrome $d$ level is significantly lower in the pss $A$ mutant strain and the activity of the peripheral NADH dehydrogenase II is strongly reduced (Mileykovskaya and Dowhan, 1993). Considering the diversity and variability of the E. coli respiratory chains, the significance of PE in their assembly, functioning and organization remains unclear. 
Finally, anionic lipids and CL in particular are required for proper functioning of Secdependent protein translocation across the cytoplasmic membrane (Lill et al., 1990; Gold et al., 2010; Dalal et al., 2012), such as cytochromes $c$ which play an active role in respiratory chains (Azai et al., 2010; Simon et al., 2011). Such observation is supported by the fact that suppressor mutations compensating for the absence of SecG were found in $p g s A$, involved in PG and CL biosynthesis (Suzuki et al., 1999). Interestingly, protein translocation by the Tat pathway capable of exporting tightly folded substrates is also severely affected by either pss or pgsA mutations (Mikhaleva et al., 1999). Again, a number of components of prokaryotic respiratory chains rely on the Tat pathway for their correct localization in the periplasm as exemplified by metalloproteins such as molybdoenzymes or hydrogenases (Berks et al., 2003; Palmer et al., 2005; Bock et al., 2006). Therefore, lipids should be considered as key determinants for supramolecular organization of the prokaryotic respiratory chains as they can affect the translocation, the topology of integral membrane proteins, the structure, the function and the oligomerization of respiratory complexes.

An important parameter when considering the amount of a given lipid species such as CL required for tuning the structure, functionality and ultimately the supramolecular organization of respiratory complexes in a prokaryotic cell is its cellular localization ie spatial parameter. During the last decade, our view on the organization of the bacterial cytoplasmic membrane has dramatically changed with the observation of distinct subdomains differing in their lipid and protein composition. For extensive reviews on bacterial cell compartimentation the reader is referred to (Gitai, 2005; Matsumoto et al., 2006; Huang and Ramamurthi, 2010; Rudner and Losick, 2010). Accordingly, the cytoplasmic membrane has to be seen as an inhomogeneous and highly dynamic structure with defined membrane microenvironments (Jacoby and Young, 1988; Vanounou et al., 2003; Engelman, 2005; Matsumoto et al., 2006; Mileykovskaya and Dowhan, 2009; Li and Young, 2012). Most interestingly, CL-enriched membrane domains were mostly observed at the cell poles and at the septal region of actively dividing bacterial cells such as E. coli (Mileykovskaya and Dowhan, 2000), B. subtilis (Kawai et al., 2004; Kawai et al., 2006), Pseudomonas putida (Bernal et al., 2007) and Mycobacterium species (Maloney et al., 2011). In contrast, polar and septal localization of CL was lost in stationary phase grown cells of M. smegmatis supporting a different lipid distribution (Maloney et al., 2011). How to explain such uneven localization of CL in rod-shaped bacteria? At first, the conical shape of CL with a headgroup cross-sectional area much smaller than that of its lipid tails supporting a curvature trend is an essential parameter for CL microdomain formation (Huang et al., 2006; Huang and Ramamurthi, 2010). In particular, such conical shape makes it 
favorable to be specifically enriched at negatively curved regions of the inner leaflet of the bacterial membranes. Furthermore, physical models anticipate that, in rod-shaped bacteria, the bacterial cell wall constrains the cytoplasmic membrane to produce finite-sized clusters of high-curvature lipids such as CL which will spontaneously and stably localize to the cell poles and at the septal region (Huang et al., 2006). Besides, such cell wall-mediated lipid phase separation requires a critical amount of CL to produce domains which will form a lattice (Mukhopadhyay et al., 2008). Additional support to this model came from a recent study showing that integrity of the cell wall peptidoglycan is required for maintenance of the lipid domains in B. subtilis (Muchova et al., 2011). Most importantly, these anionic lipid domains formed in a wide temporal and spatial scale may in turn play an important role in subcellular protein localization (Romantsov et al., 2010).

Overall, we surmise that varying the amount of CL in the prokaryotic cell temporally and spatially can provide not only an elegant, mechanistically simple way of turning on or off the activity of respiratory complexes but also can influence the higher-organization of the respiratory chain depending on the energy demand $<$ Figure $3>$.

\subsection{Proteins}

Aside from the view where lipid-lipid interactions direct a compartmentalization of a given subset of proteins displaying a high affinity for those lipids, membrane proteins can directly influence the surrounding lipids, the so-called lipid annulus and thus the formation of surrounding lipid domains (Anderson and Jacobson, 2002; Lee, 2011b; Lee, 2011a). A more accurate view is by considering a mutual impact of lipid-protein interactions on the lateral organization of biological membranes (Poveda et al., 2008; Lingwood and Simons, 2010; Groger et al., 2012). Furthermore, membrane proteins appear to diffuse in a concerted manner with numerous lipids around them, predicting only a few if any free lipids due to molecular crowing in membranes (Niemela et al., 2010). In this context, oligomerization of membrane proteins such as respiratory complexes can represent an important factor in lipid domain formation (Figure 3).

Apart from influencing the surrounding lipids and participating to lipid domain formation, one may ask whether proteins other than respiratory complexes themselves can be determinants for supramolecular organization of the prokaryotic respiratory chains. Recently, three groups have reported simultaneously the identification of the first protein factor necessary for supercomplex assembly and stability in yeast (Chen et al., 2012; Strogolova et al., 2012; 


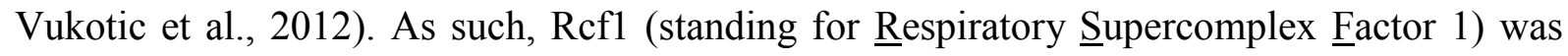
shown to be not only a stable component of mitochondrial supercomplexes $\mathrm{III}_{2} \mathrm{IV}_{1}$ or $\mathrm{III}_{2} \mathrm{IV}_{2}$ but also to be required for their formation. Rcf1 ( $\sim 20 \mathrm{kDa})$, an integral membrane protein of the mitochondrial inner membrane with two predicted transmembrane helices, is a member of the hypoxia inducible gene 1 (HIG1) family and is conserved in eukaryotes and in $\alpha$ proteobacteria. In human and mouse, two variants of Rcfl were found and are notably expressed in metabolically-active murine tissues such as kidney, heart or liver (Vukotic et al., 2012). The corresponding gene is up-regulated by stresses in the environment such as low oxygen or glucose conditions (Denko et al., 2000; Wang et al., 2006). Rcfl appears to be associated with components of complex III and IV, independently of the assembly state of the other, the tighter interaction being observed with the COX3 subunit of complex IV. Detailed analysis of the high-resolution cryo-electron microscopy reconstructions of the bovine $\mathrm{I}_{1} \mathrm{III}_{2} \mathrm{IV}_{1}$ supercomplex allowed to position the COX3 subunit at the contact interface between complexes III and IV (Althoff et al., 2011; Dudkina et al., 2011). Therefore, Rcfl may partly occupy the gap clearly visible in the membrane region between complexes III and IV (Chen et al., 2012; Vukotic et al., 2012). Finally, the most prominent observation is the phenotype in Rcf1-deleted cells. Apart from an impaired respiration, strong reduction of the stability of the supercomplexes and elevated oxidative stress are reported (Chen et al., 2012; Vukotic et al., 2012). A specific loss of complex IV activity likely due to impaired assembly is also observed in absence of Rcf1. Vukotic et al (Vukotic et al., 2012) unexpectedly reported that complex IV exists in different subtypes depending on the presence of COX13 peripheral subunit, a condition fulfilled by Rcf1. The absence of Rcf1 which precludes assembly of COX13 with the core subunits of complex IV still permits the association of a subset of complexes IV with complexes III. Finally, Rcf1 physically interacts with the ADP/ATP carrier (Aac2) also known to associate with the respiratory supercomplexes (Chen et al., 2012; Strogolova et al., 2012). As Rcf1 or CL, the absence of Aac2 results in severe destabilization of the supercomplexes (Zhang et al., 2002; Claypool et al., 2008; Dienhart and Stuart, 2008). Overall, Rcf1 should be seen as a protein factor necessary for supercomplex assembly and stability. Furthermore, by controlling the insertion of additional subunits such as COX13, Rcfl fine-tunes the activity of complex IV and of the corresponding supercomplexes preventing reactive oxygen species production. Considering the higher expression level of Rcf1 under hypoxia and its tissue specific pattern of expression, it may well be that Rcf1 influences the level and nature of respiratory supercomplexes in response to environmental or metabolic cues. In this context, plant mitochondrial supercomplexes were reported to be 
affected by hypoxia and a concomitant drop in $\mathrm{pH}$ (Ramirez-Aguilar et al., 2011). In particular, complex I dissociates from the supercomplex leaving out $\mathrm{III}_{2}$-IV subcomplexes which likely favors their functioning with the alternative NADH dehydrogenases. Considering the reported growth-dependent variation of yeast supercomplexes composition (Wittig and Schagger, 2009), a change in supercomplex composition can thus be perceived as a regulatory mechanism allowing living organisms to cope with varying environmental signals.

Interestingly, Rcf1 homologs are found in $\alpha$-proteobacteria considered to be the progenitors of the mitochondria through endosymbiosis. However, their actual function in bacteria is currently unknown. Supercomplexes made up of the cytochrome $b c_{1}$ complex with a $a a_{3}$-type or $c b b_{3}$-type cytochrome $c$ oxidase have been reported in $P$. denitrificans and B. japonicum, belonging to the $\alpha$-proteobacteria phylum (Berry and Trumpower, 1985; Keefe and Maier, 1993; Stroh et al., 2004). Owing to their small size $(\sim 10 \mathrm{kDa})$ and high hydrophobic character, the Rcf1 homologs in P. denitrificans and B. japonicum may well be undetected or lost during purification. Genome-wide transcriptome analysis has been conducted in $B$. japonicum under different growth conditions and allows inferring the expression pattern of the Rcf1 homolog corresponding gene (blr1375) (Pessi et al., 2007). One may hypothesize that Rcf1 in B. japonicum is also required for association of the cytochrome $b c_{1}$ complex with the $c b b_{3}$-type cytochrome $c$ oxidase and as such be expressed under conditions where the supercomplex has been isolated. As compared with genes encoding subunits of each respiratory complexes, the Rcfl-encoding gene is constitutively expressed which does not preclude it as being present under conditions where the supercomplex is formed (Pessi et al., 2007). Unfortunately, transcriptomic data are not available in P. denitrificans.

At this stage, one may suggest that proteic factors, in addition to specific lipids such as CL, influence the formation of supercomplexes in prokaryotes based on the recent discoveries made in yeast (Figure 3) (Chen et al., 2012; Strogolova et al., 2012; Vukotic et al., 2012). To date, Rcfl homologs with yet unknown functions are only present in $\alpha$-proteobacteria. What is then the situation in other bacteria or in archaea harboring a cytochrome $c$ oxidase? If the utmost importance of Rcfl in eukaryotes is to regulate the complex IV activity in response to environmental cues through a dynamic association with the supercomplexes, what would be the function of Rcfl in prokaryotes where several respiratory chains can coexist? In eukaryotes, Rcf1 regulates the functioning of the whole mitochondrial aerobic respiratory chain by fine-tuning the activity of the last key enzyme. In prokaryotes where cytochome $c$ oxidase can coexist with alternative terminal oxidases such as quinol oxidases, all of them 
reducing molecular oxygen in water, a similar function of the Rcf1 homologs is much less evident.

Intriguingly, CtaF $(19 \mathrm{kDa})$ with two transmembrane helices is considered to be the fourth subunit of the $a a_{3}$-type cytochrome $c$ oxidase of $C$. glutanicum (Niebisch and Bott, 2003) with homologs present in all members of the actinobacteria phylum i.e. gram positive bacteria with high guanine and cytosine content. CtaF co-purified with isolated $a a_{3}$-type cytochrome $c$ oxidase as well with the cytochrome $b c_{1}-a a_{3}$ supercomplex in $C$. glutanicum but not with the $b c_{1}$ complex (in contrast with Rcf1 in yeast) (Niebisch and Bott, 2003). CtaF is essential for the formation of an active $a a_{3}$-type cytochrome $c$ oxidase but is probably not essential for its catalytic activity as loss of CtaF during purification is not associated with a modification of the activity (Sakamoto et al., 2001). Finally, a hitherto uncharacterized protein of $17 \mathrm{kDa}$ copurified with the cytochrome $b c_{1}-a a_{3}$ supercomplex but was absent from the isolated complexes (Niebisch and Bott, 2003). While it remains open whether this protein is required for supercomplex formation and/or stability, these two examples indicate that proteins sharing analogies with Rcfl might be more widespread than originally thought.

\subsection{Subcellular localization}

Far from the pioneering mosaic model depicted by Singer and Nicolson (Singer and Nicolson, 1972), the actual view is that lipids and proteins are nonhomogeneously distributed in membranes. Membrane components are organized in small domains or clusters of highly variable composition and length-scale. Eukaryotic cells subdivised into many subcellular compartments are known to be highly organized at a molecular level. By the same token, prokaryotic cells also possess large and distinct structures which are located in specific sites such as chemoreceptors or cell appendages such as pili, flagella, stalks. Moreover, their uneven location is often intimately linked to their biological function. Apart from these large structures, recent advances in fluorescence live-cell imaging have allowed not only to expand the range of molecules (protein, lipid, chromosome and RNA) located in distinct subcellular locations but also to introduce a dynamic parameter both at the spatial and time level (see for reviews, (Shapiro et al., 2009; Kusumi et al., 2010; Rudner and Losick, 2010; Amster-Choder, 2011). At this stage, dynamic subcellular localization should be seen as a trait intimately linked to the biological function allowing control of fundamental processes such as prokaryotic cell development, chromosome partitioning or motility. Despite the accumulated findings on the subcellular localization of molecules in bacteria, much remain to be 
understood in terms of the mechanisms involved for such spatial and temporal distribution resulting in various models to explain specific localization of a protein within the bacterial cell (Mignot and Shaevitz, 2008; Shapiro et al., 2009; Huang and Ramamurthi, 2010; Ramamurthi, 2010; Rudner and Losick, 2010). In this section, we will question the existence of a non-uniform distribution of respiratory complexes which intuitively may have an impact in terms of their supramolecular organization.

Approximately one third of all proteins are integral membrane proteins, most of them being components of multimeric respiratory complexes. Interestingly, a high protein concentration within the membrane is considered to favor the colocalization of proteins of a same family in same clusters (Meilhac and Destainville, 2011) with a significant contribution of the transmembrane helices for such lipid/protein partitioning (Domanski et al., 2012). Clusterization and molecular crowding may thus favor interactions between membrane proteins which would be relevant for respiratory processes. Consequently, protein concentration is seen as a determinant for supercomplex organization in mitochondria (Lenaz and Genova, 2010). Illustration of the intimate relationships between molecular crowding and supercomplex formation can be taken with the thylakoid membranes which display a highly dense protein-packing (Dudkina et al., 2006a; Sturgis and Niederman, 2008; Woronowicz et al., 2011; Kouril et al., 2012). The immediate consequence for high protein concentration is a dramatic reduction of the lateral diffusion processes (Kirchhoff et al., 2008). Thereof, it has recently been proposed that supercomplex formation is part of a strategy aiming to minimize such problems (Kirchhoff, 2008b; Kirchhoff, 2008a). In the mitochondrial inner membrane, components of the respiratory chain are also densely packed resulting in a short average distance between complexes which may favor supercomplex formation.

Spatial heterogeneity can contribute further to the biological functions by concentrating a subset of defined proteins in a membrane domain, thus facilitating interaction as opposite with a situation where the membrane proteins are randomly dispersed. For instance, the cytochrome $b d$-I, one of the three terminal oxidases of the aerobic respiratory chain of $E$. coli (Figure 2) was reported to be unevenly distributed within the inner membrane using real-time fluorescence microscopy (Lenn et al., 2008b). Indeed, this respiratory complex is concentrated in mobile patches with an average number of molecules of 80 . Similarly, the succinate dehydrogenase and the ATP synthase from B. subtilis were shown to behave similarly within discrete clusters (Johnson et al., 2004). Apart from those fluorescence imaging approaches, large-scale proteomic approaches aiming at differentiating membrane proteins localized at the poles versus the lateral walls of $E$. coli cells have provided valuable 
information relative to the localization of several respiratory complexes (Lai et al., 2004; Li and Young, 2012). The ATP synthase was either found enriched in minicells issued from the poles (Lai et al., 2004) or evenly distributed (Kitagawa et al., 2005; Li and Young, 2012). Among the 15 dehydrogenases and 11 terminal oxidases from E. coli (Figure 2), only a subset was detected likely due to experimental pitfalls or expression conditions. With the exception of the nitrate reductase $\mathrm{Z}$ found exclusively at the pole, NDH-I, succinate dehydrogenase, formate dehydrogenase $\mathrm{O}$, glycerol 3-phosphate dehydrogenase and cytochromes $b o_{3}$ or $b d$-I were uniformly distributed ( $\mathrm{Li}$ and Young, 2012). In the latter case, it should be mentioned that it cannot be ruled out that those complexes are present in discrete patches dispersed throughout the cell. Aside from providing for the first time a systematic list of pole-localized proteins in E. coli, much remain to be done to confirm such distribution. Considering the diversity of respiratory complexes and prokaryotic organisms, these few reports are far from being sufficient to give an accurate estimation of the situation. However, they allow to question whether subcellular localization of respiratory complexes is at play, at least in E. coli cells. Based on the observation of mobile patches of cytochrome $b d$-I in E. coli, Mullineaux's group has hypothesized that compartmentalization of processes related to respiration may occur within the bacterial cytoplasmic membrane leading to the notion of respirazones, i.e. specialized compartments dedicated to respiration (Figure 3) (Lenn et al., 2008a). This hypothesis recalls the "compartment" model depicted in Figure 1C for the mitochondrial respiratory chain. Other respiratory complexes would then be present in cytochrome $b d-\mathrm{I}$ clusters. The observation that specific lipids such as CL tend to cluster at the cell poles and at the division septum (Nishibori et al., 2005; Mileykovskaya and Dowhan, 2009) and that several respiratory complexes strongly and specifically bind CL (Arias-Cartin et al., 2012) leads to question whether CL can be used as a cue for their spatial distribution. For instance, the nitrate reductase A complex from E. coli was shown to shift from an inactive to an active state upon CL binding (Arias-Cartin et al., 2011). This observation suggests that both states may coexist within the same bacterial cell, their interconversion being related to CL avaibility and or spatial distribution. A direct demonstration of such hypothesis awaits further studies.

By virtue of the branched character of the prokaryotic respiratory chains, uneven distribution of a given respiratory complex in the cell has to be seen in regards to the localization of the other components of the respiratory chain. Protein mobility thus appears to be a key parameter in the description of such processes. It is well established that the membrane protein lateral mobility depends on membrane fluidity and more on the number of their transmembrane helices than on the size of the diffusing species (Kumar et al., 2010). The lateral mobility of 
proteins can be further modified by molecular crowding (Frick et al., 2007; Ramadurai et al., 2009) as well as by interactions with membrane microdomains and the cytoskeleton (Lenne et al., 2006; Lopez and Kolter, 2010; Domanov et al., 2011; Kusumi et al., 2011). One may question whether the change in mobility of a given respiratory complex can provide a regulatory pathway in the respiratory process as a whole but more works needs to be done to answer this question.

Recently, Zhuang et al have proposed that prokaryotic cellular metabolism is fundamentally constrained by the finite cytoplasmic membrane surface area available for protein expression (Zhuang et al., 2011). Indeed, their rationale is that a competition exists between substrate transporters and respiratory enzymes since both of them are localized on the cytoplasmic membrane. As such, increased substrate uptake rates can only be achieved through a decrease in the respiratory rate obtained by processing the substrate through the fermentative pathways, which are not catalyzed by transmembrane proteins, for ATP production. Despite the fact that a number of respiratory complexes were not taken into consideration in their flux balance analysis (FBA), such proposal strengthens the importance for molecular crowding in the membrane and the necessity to optimize its occupancy. Having this thought-provoking hypothesis in consideration, specific distribution of a given subset of respiratory complexes with their ultimate physical association in supercomplexes may be beneficial in terms of membrane occupancy (Figure 3). The simplest way to achieve metabolic transitions would then be a random redistribution of the complexes. Finally, morphological alterations during prokaryotic cell development likely participate to this phenomenon by influencing the membrane surface area to volume ratio.

\section{Implications of supramolecular organization}

As mentioned in the introduction, the respiratory flexibility of the mitochondria in eukaryotes is rather poor. Apart from some flexibility at the level of electron input with the participation of various transmembrane or membrane-associated complexes, none is present at the level of electron output which involves the cytochrome $c$ oxidase for oxygen reduction. An exception may be seen with the reported existence of anaerobic mitochondria in protists and metazoan using nitrate or fumarate as alternative electron acceptors to oxygen (Tielens et al., 2002; Müller et al., 2012). On the opposite, bacteria and archaea are characterized by an extreme respiratory flexibility with the use of a diverse range of electron acceptors other than oxygen including the nitrogen oxides, transition metals, metalloid oxyanions, organic N-oxides, 
elemental sulphur and sulphur oxyanions or halogenated organics (see for instance (Berks et al., 1995; van de Pas et al., 1999; Cabello et al., 2004; Lovley et al., 2004; McCrindle et al., 2005; Stolz et al., 2006; Zumft and Kroneck, 2007; Cramm, 2009; Arai, 2011; Bird et al., 2011). Such respiratory flexibility found in a wide range of prokaryotes allows them to cope with extreme environmental conditions (temperature, salinity, pressure, oxygen...) or in many pathogenic bacteria (Kelly, 1998; Boshoff and Barry, 2005; Kern and Simon, 2009; Danielli and Scarlato, 2010)). At a molecular level, the flexibility arises from a tight and modulated control of the expression of different terminal oxido-reductases allowing the organism to adapt quickly in a rapidly changing environment (Richardson, 2000). Furthermore, within the same organism, several biochemically distinct enzymes catalyzing essentially the same reaction may coexist. This is exemplified, in Escherichia coli, by three terminal oxidases of the aerobic respiratory chain coupling quinol oxidation to oxygen reduction (cytochromes $\mathrm{bo}_{3}$, $b d$-I and $b d$-II) (Figure 2) (Poole and Cook, 2000). These enzymes are characterized by distinct affinities towards oxygen as well as slightly different expression patterns (Borisov and Verkhovsky, 2009; Borisov et al., 2011). In principle, thanks to their ability to the common use of quinones as redox mediators, any respiratory dehydrogenase is able to react with any terminal oxido-reductase. Nevertheless, the situation appears more complicate due to differential expression patterns and different affinities towards various types of quinones.

In contrast with the detailed knowledge on individual respiratory enzymes, the enzymatic composition of prokaryotic electron transport chains as well as the involved protein-protein interactions are less well understood. Physical association of respiratory complexes in the form of so-called supercomplexes has been reported in few instances both in bacteria and archaea raising the question of the universality of such supramolecular organization. Additionally, does such observation provide an argument towards the "solid state" model (Figure 1B) which implies association of complexes and confinement of the mobile carriers? On the opposite, the "liquid state" model implies free diffusion of the carriers that randomly interact with their protein partners (Figure 1A). Most importantly, besides the question of the degree of restricted mobility of mobile electron carriers, we would like in the following to focus on what are the implications for such organization. Owing to the well-reported supramolecular organization of the mitochondrial respiratory chain in eukaryotes, several hypotheses have been formulated to explain the consequences of such arrangement and can be herein further discussed in the context of prokaryotes.

At first, supercomplexes may increase the stability of individual complexes. Indeed, genetic alterations leading to a loss of complex III in mitochondria are known to prevent 
supercomplex formation and led to a secondary loss of complex I (Acin-Perez et al., 2004; Schagger et al., 2004; D'Aurelio et al., 2006). Conversely, stability of complex III is not influenced by the absence of complex I. However, the first indication of such gain of stability of complex I upon association within supercomplexes has been obtained in the $\alpha$ proteobacterium $P$. denitrificans (Stroh et al., 2004). To date, no evidence has been reported for a similar situation in other complexes involved in supercomplexes in prokaryotes. Most of the individual components of prokaryotic supercomplexes have been previously isolated without reported instability. Furthermore, in E. coli devoid of complexes III and IV, the complex I is a fully stable entity (Schneider et al., 2008). Recently, the Ugalde's group has provided a molecular description of the mitochondrial respirasome assembly allowing to explain the requirements for complex I stability (Moreno-Lastres et al., 2012). Respirasome assembly appears to occur in a multistep fashion and to involve a complex I assembly intermediate acting as a scaffold for subsequent incorporation of complexes III and IV subunits. This situation would be restricted to mammals as plants are reported to contain isolated complex I from III-IV supercomplexes (Ramirez-Aguilar et al., 2011). Overall, role of supramolecular organization in prokaryotes in stability and assembly remains open and cannot be ruled out at this stage.

A second prominent role of supercomplexes has been proposed at a kinetic level through substrate channeling and confinement of the mobile carriers (quinones and cytochrome $c$ ). While the physiological relevance of supercomplexes can be deduced from the reported variation in their abundance and composition in response to physiological changes (Gomez et al., 2009; Helbig et al., 2009; Rosca and Hoppel, 2009; Wittig and Schagger, 2009; Frenzel et al., 2010; Gomez and Hagen, 2012) or cell differentiation (Hofmann et al., 2012), very little functional evidence is available. Isolated respirasomes were reported to promote electron transfer from NADH or succinate to oxygen supportive of their functionality (Acin-Perez et al., 2008). Using isolated yeast mitochondria, Boumans et al reported that the respiratory chain functions as a functional unit since neither quinone nor cytochrome $c$ exhibits pool behavior (Boumans et al., 1998). Recently, the first analysis in intact yeast cells of the functional relevance of supercomplexes has led to reconsider substrate channeling as the result of supramolecular organization of the respiratory chain (Trouillard et al., 2011). In particular, the authors demonstrate that the respiratory chain in intact cells essentially behaves as a homogenous system showing that cytochrome $c$ is not confined to supercomplexes on the time scale of hundreds of milliseconds. Apart from the reported existence of III-IV supercomplexes and the participation of additional proteins for their physical association such 
as Rcfl, this study strengthens the fact that such organization has no significant influence of the mobility of the cytochrome $c$. The situation is less clear concerning quinones. In $P$. denitrificans, isolation of the supercomplex made up of the complexes I, III and IV has shown a strong enrichment in ubiquinone (Stroh et al., 2004). Such observation raises the question of the existence of a compartmented quinone pool associated with the supercomplex. If a clear answer to this question awaits further studies, the Lenaz's group has shown that supercomplex formation in bovine heart mitochondrial particles enhances the electron transfer above that envisioned with a homogenous pool (Bianchi et al., 2003). Another functional consequence of substrate channeling, if any, is the differential activity of isolated complexes as compared when part of supercomplexes. Again, to our knowledge, only few information is available. However, a clear answer to this question is difficult due to the known structural and functional importance of lipids for respiratory complexes (see section 3.1) which are lost during isolation of the complexes (Lee, 2004; Hunte, 2005; Bogdanov et al., 2008; AriasCartin et al., 2012).

A number of studies in eukaryotes have reported a role of mitochondrial supercomplex organization on limiting ROS production which could be seen as another functional consequence of their existence. Hence, several pathological states which lead to mitochondrial dysfunction such as reducing the amount of CL (Barth syndrome, ischemia, hypothyroidism, heart failure, aging) are associated with a decrease in supercomplexes, in the activity of a number of respiratory complexes and often linked with the concomitant increase of ROS, byproduct of oxidative phosphorylation (McKenzie et al., 2006; Chicco and Sparagna, 2007; Frenzel et al., 2010; Acehan et al., 2011b; Gomez and Hagen, 2012). Furthermore, ROSdriven peroxidation of $\mathrm{CL}$ molecules tightly interacting with the respiratory complexes impacts their activity (Petrosillo et al., 2003a). Recently, the absence of the Rcf1 protein factor was reported to be associated not only with a strong reduction of the stability of the supercomplexes but with impaired respiration and elevated oxidative stress (Chen et al., 2012; Vukotic et al., 2012). These results clearly support an important role of supercomplex formation in preventing adventitious production of ROS. One has to keep in mind that ROS production is considered to occur at specific cavities of a number of respiratory complexes at which quinones bound and their reactive semiquinone radical intermediates stabilized during the course of the one-electron transfer reaction to or from metal centers. Each of the three mitochondrial respiratory complexes interacting with quinones was reported to be associated with superoxide production upon functioning (Muller et al., 2002; Grivennikova and Vinogradov, 2006; Lenaz et al., 2006; Muller et al., 2008; Treberg et al., 2011; Quinlan et al., 
2012). A prominent example is the complex III functioning with a Q-cycle which involves formation of semiquinone intermediates (Cape et al., 2006; Cape et al., 2007). To our knowledge, such causal connection between supercomplexes organization and limiting ROS generation in prokaryotes has not been evaluated. Interestingly, superoxide production in the periplasm of $E$. coli has been linked to the redox state of menaquinone ie to the functioning of the respiratory chain (Korshunov and Imlay, 2006). While the source of superoxide production remains unclear, it may well be that semiquinone stabilization by respiratory complexes accounts for it. If supercomplexes of the aerobic respiratory chain are at play in $E$. coli as reported by Sousa et al (Sousa et al., 2011), does such a supramolecular organization of the respiratory chain has an influence on the level of superoxide production? Thereof, how to explain superoxide production under normal functioning of the aerobic respiratory chain? Finally, studies may have to be conducted during the aerobic-anaerobic transition during which a number of various respiratory complexes having the ability to stabilize reactive semiquinone intermediates are present and may be part of transient supercomplexes.

Finally, subcellular localization of respiratory complexes and their supramolecular assemblies can have additional implications both at a functional and cellular level. Spatial segregation of the respiratory complexes and of the ATP synthase in distinct subdomains of the cristae membrane was reported in several eukaryotes (Davies et al., 2011). Such spatial arrangement of complexes serving as proton sources apart but in close proximity of the ATP synthase serving as proton sink was proposed to ensure optimal conditions for efficient ATP synthesis (Figure 1C). Moreover, simulation of the electric field on membrane surface indicates a higher charge density and hence a higher proton concentration in areas of sharp membrane curvature such as the one observed at the cristae edges thus resulting in a stronger $\Delta \mathrm{pH}$ contribution to the pmf in highly curved regions (Strauss et al., 2008). How can this be translated in bacteria? Poles of rod-shaped bacteria are known to compartmentalize proteins which recognize the negative membrane curvature as a cue or a specific enrichment in CL known to cluster and concentrate at the poles and at the septum of dividing cells (Nishibori et al., 2005; Mileykovskaya and Dowhan, 2009). In addition, a number of respiratory complexes are reported to require CL for functionality. Thereof, do we have to consider that respiratory complexes which depend on CL for optimal activity are polarly localized? Does their functioning in such highly curved membrane domains participate to a higher local proton concentration? Does supercomplex formation in subcellular domains favor the overall yield? Is this exploited by a similar localization of the ATP synthase? In fact, the general question of respiratory complexes localization in bacterial cells has only been partly addressed until now. 
Having in mind the high flexibility character of the respiratory systems in prokaryotes, the use of real-time fluorescence imaging should allow to provide not only spatial but also temporal information of valuable importance.

\section{Concluding remarks and Future directions}

With more than 2500 prokaryotic genomes available, a huge database is now accessible to the scientific community to explore the biochemical diversity of mechanisms transducing electron transfer to an electrochemical gradient subsequently used by a number of essential processes among which ATP synthesis. Major goals in the future studies will be to characterize at the cellular level the distribution of respiratory complexes and their physical association. Considering the inherent metabolic flexibility of a number of prokaryotes, distribution and composition of the supramolecular assemblies should be seen in regards to environmental signals $i e$ in response to the metabolic demand. In this context, invaluable information in terms of composition and dynamics of respiratory chains would be provided by real-time fluorescence microscopy. In contrast with electron microscopy approaches, light microscopes allow for dynamic studies of live cells which combined with super-resolution light microscopic methods allow to overcome diffraction limited resolution and the observation of membrane protein clusters (Greenfield et al., 2009; Lang and Rizzoli, 2010). At a nanoscale range, studies about membrane microdomains and specific protein-protein or protein-lipid contacts can benefit from recent advances in FRET imaging (Loura and Prieto, 2011) or timeresolved fluorescence microscopy (Smith, 2012). Overall, these approaches would provide a comprehensive physical description of the organization of the respiratory chains and of the dynamics of their components in live cell membranes.

At a molecular level, of special interest is the identification of the low molecular weight molecules associated with the isolated supercomplexes including lipids, quinones or even small-size and hydrophobic proteins. Until now, these molecules often escaping detection were not sufficiently considered. With the recent advances in the mass spectrometry of membrane proteins, these hurdles are overtaken and novel possibilities are now offered to determine subunit stoichiometry or dissect lipid-binding properties of respiratory complexes (Barrera and Robinson, 2011). Ultimately, we may envision the use of high-throughput livecell imaging approaches to identify determinants for supercomplexes formation.

In this review, we discuss many potential determinants for supramolecular organization of the prokaryotic respiratory chains. In this context, the inclusion of spatiotemporal aspects outlined 
above is critical. However, functional implications of such organization are largely hypothetical and could only be addressed through concerted efforts from different disciplines on a larger group of prokaryotic systems.

\section{Acknowledgments}

We would like to thank our collaborators and colleagues for fruitful discussions. We thank the CNRS, the Aix-Marseille University and the Agence Nationale pour la Recherche for their funding and continuous support.

\section{References}

Acehan, D., Malhotra, A., Xu, Y., Ren, M., Stokes, D. L. and Schlame, M. (2011a).Cardiolipin affects the supramolecular organization of ATP synthase in mitochondria Biophys $J$ 100, 2184-92.

Acehan, D., Vaz, F., Houtkooper, R. H., James, J., Moore, V., Tokunaga, C., Kulik, W., Wansapura, J., Toth, M. J., Strauss, A. and Khuchua, Z. (2011b).Cardiac and skeletal muscle defects in a mouse model of human Barth syndrome J Biol Chem 286, 899908.

Acin-Perez, R., Bayona-Bafaluy, M. P., Fernandez-Silva, P., Moreno-Loshuertos, R., PerezMartos, A., Bruno, C., Moraes, C. T. and Enriquez, J. A. (2004).Respiratory complex III is required to maintain complex I in mammalian mitochondria Mol Cell 13, 805-15.

Acin-Perez, R., Fernandez-Silva, P., Peleato, M. L., Perez-Martos, A. and Enriquez, J. A. (2008).Respiratory active mitochondrial supercomplexes Mol Cell 32, 529-39.

Althoff, T., Mills, D. J., Popot, J. L. and Kuhlbrandt, W. (2011).Arrangement of electron transport chain components in bovine mitochondrial supercomplex I1III2IV1 EMBO J 30, 4652-64.

Amster-Choder, O. (2011).The compartmentalized vessel: The bacterial cell as a model for subcellular organization (a tale of two studies) Cell Logist 1, 77-81.

Anderson, R. G. and Jacobson, K. (2002).A role for lipid shells in targeting proteins to caveolae, rafts, and other lipid domains Science 296, 1821-5.

Arai, H. (2011).Regulation and Function of Versatile Aerobic and Anaerobic Respiratory Metabolism in Pseudomonas aeruginosa Front Microbiol 2, 103. 
Arias-Cartin, R., Grimaldi, S., Arnoux, P., Guigliarelli, B. and Magalon, A. (2012).Cardiolipin binding in bacterial respiratory complexes: Structural and functional implications Biochim Biophys Acta.

Arias-Cartin, R., Grimaldi, S., Pommier, J., Lanciano, P., Schaefer, C., Arnoux, P., Giordano, G., Guigliarelli, B. and Magalon, A. (2011).Cardiolipin-based respiratory complex activation in bacteria Proc Natl Acad Sci U S A 108, 7781-6.

Asai, Y., Katayose, Y., Hikita, C., Ohta, A. and Shibuya, I. (1989).Suppression of the lethal effect of acidic-phospholipid deficiency by defective formation of the major outer membrane lipoprotein in Escherichia coli J Bacteriol 171, 6867-9.

Azai, C., Tsukatani, Y., Itoh, S. and Oh-oka, H. (2010).C-type cytochromes in the photosynthetic electron transfer pathways in green sulfur bacteria and heliobacteria Photosynth Res 104, 189-99.

Barrera, N. P. and Robinson, C. V. (2011).Advances in the mass spectrometry of membrane proteins: from individual proteins to intact complexes Annu Rev Biochem 80, 247-71.

Berg, B. L. and Stewart, V. (1990).Structural genes for nitrate-inducible formate dehydrogenase in Escherichia coli K-12 Genetics 125, 691-702.

Berks, B. C., Ferguson, S. J., Moir, J. W. and Richardson, D. J. (1995).Enzymes and associated electron transport systems that catalyse the respiratory reduction of nitrogen oxides and oxyanions Biochim Biophys Acta 1232, 97-173.

Berks, B. C., Palmer, T. and Sargent, F. (2003).The Tat protein translocation pathway and its role in microbial physiology Adv Microb Physiol 47, 187-254.

Bernal, P., Munoz-Rojas, J., Hurtado, A., Ramos, J. L. and Segura, A. (2007).A Pseudomonas putida cardiolipin synthesis mutant exhibits increased sensitivity to drugs related to transport functionality Environ Microbiol 9, 1135-45.

Berry, E. A., Guergova-Kuras, M., Huang, L. S. and Crofts, A. R. (2000).Structure and function of cytochrome bc complexes Annu Rev Biochem 69, 1005-75.

Berry, E. A. and Trumpower, B. L. (1985).Isolation of ubiquinol oxidase from Paracoccus denitrificans and resolution into cytochrome bc1 and cytochrome c-aa3 complexes $J$ Biol Chem 260, 2458-67.

Bertero, M. G., Rothery, R. A., Palak, M., Hou, C., Lim, D., Blasco, F., Weiner, J. H. and Strynadka, N. C. (2003).Insights into the respiratory electron transfer pathway from the structure of nitrate reductase A Nat Struct Biol 10, 681-7. 
Beyer, K. and Nuscher, B. (1996).Specific cardiolipin binding interferes with labeling of sulfhydryl residues in the adenosine diphosphate/adenosine triphosphate carrier protein from beef heart mitochondria Biochemistry 35, 15784-90.

Bianchi, C., Fato, R., Genova, M. L., Parenti Castelli, G. and Lenaz, G. (2003).Structural and functional organization of Complex I in the mitochondrial respiratory chain Biofactors 18, 3-9.

Bianchi, C., Genova, M. L., Parenti Castelli, G. and Lenaz, G. (2004).The mitochondrial respiratory chain is partially organized in a supercomplex assembly: kinetic evidence using flux control analysis $J$ Biol Chem 279, 36562-9.

Bird, L. J., Bonnefoy, V. and Newman, D. K. (2011).Bioenergetic challenges of microbial iron metabolisms Trends Microbiol 19, 330-40.

Bock, A., King, P. W., Blokesch, M. and Posewitz, M. C. (2006).Maturation of hydrogenases Adv Microb Physiol 51, 1-71.

Bogdanov, M., Heacock, P. N. and Dowhan, W. (2002).A polytopic membrane protein displays a reversible topology dependent on membrane lipid composition Embo $J$ 21, 2107-16.

Bogdanov, M., Mileykovskaya, E. and Dowhan, W. (2008).Lipids in the assembly of membrane proteins and organization of protein supercomplexes: implications for lipidlinked disorders Subcell Biochem 49, 197-239.

Borisov, V. B., Murali, R., Verkhovskaya, M. L., Bloch, D. A., Han, H., Gennis, R. B. and Verkhovsky, M. I. (2011).Aerobic respiratory chain of Escherichia coli is not allowed to work in fully uncoupled mode Proc Natl Acad Sci U S A 108, 17320-4.

Borisov, V. B. and Verkhovsky, M. I. (2009). In "EcoSal: Escherichia coli and Salmonella: cellular and molecular biology" (A. Böck, ed.), vol. chapter 3.2.7. ASM press, Washington, DC.

Boshoff, H. I. and Barry, C. E., 3rd. (2005).Tuberculosis - metabolism and respiration in the absence of growth Nat Rev Microbiol 3, 70-80.

Bott, M. and Niebisch, A. (2003). The respiratory chain of Corynebacterium glutamicum $J$ Biotechnol 104, 129-53.

Boumans, H., Grivell, L. A. and Berden, J. A. (1998). The respiratory chain in yeast behaves as a single functional unit $J$ Biol Chem 273, 4872-7.

Cabello, P., Roldan, M. D. and Moreno-Vivian, C. (2004).Nitrate reduction and the nitrogen cycle in archaea Microbiology 150, 3527-46. 
Cape, J. L., Bowman, M. K. and Kramer, D. M. (2006).Understanding the cytochrome bc complexes by what they don't do. The Q-cycle at 30 Trends Plant Sci 11, 46-55.

Cape, J. L., Bowman, M. K. and Kramer, D. M. (2007).A semiquinone intermediate generated at the Qo site of the cytochrome bc1 complex: importance for the Q-cycle and superoxide production Proc Natl Acad Sci U S A 104, 7887-92.

Card, G. L. and Trautman, J. K. (1990).Role of anionic lipid in bacterial membranes Biochim Biophys Acta 1047, 77-82.

Castelle, C., Guiral, M., Malarte, G., Ledgham, F., Leroy, G., Brugna, M. and GiudiciOrticoni, M. T. (2008).A new iron-oxidizing/O2-reducing supercomplex spanning both inner and outer membranes, isolated from the extreme acidophile Acidithiobacillus ferrooxidans $J$ Biol Chem 283, 25803-11.

Catucci, L., Depalo, N., Lattanzio, V. M., Agostiano, A. and Corcelli, A. (2004).Neosynthesis of cardiolipin in Rhodobacter sphaeroides under osmotic stress Biochemistry 43, 15066-72.

Chance, B. and Williams, G. R. (1955).A method for the localization of sites for oxidative phosphorylation Nature 176, 250-4.

Chen, Y. C., Taylor, E. B., Dephoure, N., Heo, J. M., Tonhato, A., Papandreou, I., Nath, N., Denko, N. C., Gygi, S. P. and Rutter, J. (2012).Identification of a protein mediating respiratory supercomplex stability Cell Metab 15, 348-60.

Chicco, A. J. and Sparagna, G. C. (2007).Role of cardiolipin alterations in mitochondrial dysfunction and disease Am J Physiol Cell Physiol 292, C33-44.

Claypool, S. M. (2009).Cardiolipin, a critical determinant of mitochondrial carrier protein assembly and function Biochim Biophys Acta 1788, 2059-68.

Claypool, S. M., Oktay, Y., Boontheung, P., Loo, J. A. and Koehler, C. M. (2008).Cardiolipin defines the interactome of the major ADP/ATP carrier protein of the mitochondrial inner membrane $J$ Cell Biol 182, 937-50.

Corcelli, A. (2009).The cardiolipin analogues of Archaea Biochim Biophys Acta.

Cramm, R. (2009).Genomic view of energy metabolism in Ralstonia eutropha H16 J Mol Microbiol Biotechnol 16, 38-52.

Cronan, J. E., Jr. (1968).Phospholipid alterations during growth of Escherichia coli $J$ Bacteriol 95, 2054-61.

D'Aurelio, M., Gajewski, C. D., Lenaz, G. and Manfredi, G. (2006).Respiratory chain supercomplexes set the threshold for respiration defects in human mtDNA mutant cybrids Hum Mol Genet 15, 2157-69. 
Dalal, K., Chan, C. S., Sligar, S. G. and Duong, F. (2012).Two copies of the SecY channel and acidic lipids are necessary to activate the SecA translocation ATPase Proc Natl Acad Sci U S A 109, 4104-9.

Dancey, G. F. and Shapiro, B. M. (1977).Specific phospholipid requirement for activity of the purified respiratory chain NADH dehydrogenase of Escherichia coli Biochim Biophys Acta 487, 368-77.

Danielli, A. and Scarlato, V. (2010).Regulatory circuits in Helicobacter pylori : network motifs and regulators involved in metal-dependent responses FEMS Microbiol Rev 34, $738-52$.

Davies, K. M., Strauss, M., Daum, B., Kief, J. H., Osiewacz, H. D., Rycovska, A., Zickermann, V. and Kuhlbrandt, W. (2011).Macromolecular organization of ATP synthase and complex I in whole mitochondria Proc Natl Acad Sci US A 108, 141216.

de Andrade Rosa, I., Einicker-Lamas, M., Roney Bernardo, R., Previatto, L. M., MohanaBorges, R., Morgado-Diaz, J. A. and Benchimol, M. (2006).Cardiolipin in hydrogenosomes: evidence of symbiotic origin Eukaryot Cell 5, 784-7.

DeChavigny, A., Heacock, P. N. and Dowhan, W. (1991).Sequence and inactivation of the pss gene of Escherichia coli. Phosphatidylethanolamine may not be essential for cell viability $J$ Biol Chem 266, 10710.

Denko, N., Schindler, C., Koong, A., Laderoute, K., Green, C. and Giaccia, A. (2000).Epigenetic regulation of gene expression in cervical cancer cells by the tumor microenvironment Clin Cancer Res 6, 480-7.

Depalo, N., Catucci, L., Mallardi, A., Corcelli, A. and Agostiano, A. (2004).Enrichment of cardiolipin content throughout the purification procedure of photosystem II Bioelectrochemistry 63, 103-6.

Dienhart, M. K. and Stuart, R. A. (2008).The yeast Aac2 protein exists in physical association with the cytochrome bc1-COX supercomplex and the TIM23 machinery Mol Biol Cell 19, 3934-43.

Dirmeier, R., Keller, M., Frey, G., Huber, H. and Stetter, K. O. (1998).Purification and properties of an extremely thermostable membrane-bound sulfur-reducing complex from the hyperthermophilic Pyrodictium abyssi Eur J Biochem 252, 486-91.

Domanov, Y. A., Aimon, S., Toombes, G. E., Renner, M., Quemeneur, F., Triller, A., Turner, M. S. and Bassereau, P. (2011).Mobility in geometrically confined membranes Proc Natl Acad Sci U S A 108, 12605-10. 
Domanski, J., Marrink, S. J. and Schafer, L. V. (2012).Transmembrane helices can induce domain formation in crowded model membranes Biochim Biophys Acta 1818, 984-94.

Dowhan, W. and Bogdanov, M. (2009).Lipid-dependent membrane protein topogenesis Annu Rev Biochem 78, 515-40.

Dudkina, N. V., Heinemeyer, J., Keegstra, W., Boekema, E. J. and Braun, H. P. (2005).Structure of dimeric ATP synthase from mitochondria: an angular association of monomers induces the strong curvature of the inner membrane FEBS Lett 579, 5769-72.

Dudkina, N. V., Heinemeyer, J., Sunderhaus, S., Boekema, E. J. and Braun, H. P. (2006a).Respiratory chain supercomplexes in the plant mitochondrial membrane Trends Plant Sci 11, 232-40.

Dudkina, N. V., Kouril, R., Peters, K., Braun, H. P. and Boekema, E. J. (2010a).Structure and function of mitochondrial supercomplexes Biochim Biophys Acta 1797, 664-70.

Dudkina, N. V., Kudryashev, M., Stahlberg, H. and Boekema, E. J. (2011).Interaction of complexes I, III, and IV within the bovine respirasome by single particle cryoelectron tomography Proc Natl Acad Sci US A 108, 15196-200.

Dudkina, N. V., Oostergetel, G. T., Lewejohann, D., Braun, H. P. and Boekema, E. J. (2010b).Row-like organization of ATP synthase in intact mitochondria determined by cryo-electron tomography Biochim Biophys Acta 1797, 272-7.

Dudkina, N. V., Sunderhaus, S., Braun, H. P. and Boekema, E. J. (2006b).Characterization of dimeric ATP synthase and cristae membrane ultrastructure from Saccharomyces and Polytomella mitochondria FEBS Lett 580, 3427-32.

Eble, K. S., Coleman, W. B., Hantgan, R. R. and Cunningham, C. C. (1990).Tightly associated cardiolipin in the bovine heart mitochondrial ATP synthase as analyzed by 31P nuclear magnetic resonance spectroscopy J Biol Chem 265, 19434-40.

Engelman, D. M. (2005).Membranes are more mosaic than fluid Nature 438, 578-80.

Enoch, H. G. and Lester, R. L. (1975). The purification and properties of formate dehydrogenase and nitrate reductase from Escherichia coli $J$ Biol Chem 250, 6693705.

Ernst, A. M., Contreras, F. X., Brugger, B. and Wieland, F. (2010).Determinants of specificity at the protein-lipid interface in membranes FEBS Lett 584, 1713-20.

Esfahani, M., Rudkin, B. B., Cutler, C. J. and Waldron, P. E. (1977).Lipid-protein interactions in membranes: interaction of phospholipids with respiratory enzymes of Escherichia coli membrane J Biol Chem 252, 3194-8. 
Esser, L., Elberry, M., Zhou, F., Yu, C. A., Yu, L. and Xia, D. (2008).Inhibitor-complexed structures of the cytochrome bc1 from the photosynthetic bacterium Rhodobacter sphaeroides J Biol Chem 283, 2846-57.

Eubel, H., Heinemeyer, J. and Braun, H. P. (2004a).Identification and characterization of respirasomes in potato mitochondria Plant Physiol 134, 1450-9.

Eubel, H., Heinemeyer, J., Sunderhaus, S. and Braun, H. P. (2004b).Respiratory chain supercomplexes in plant mitochondria Plant Physiol Biochem 42, 937-42.

Frenzel, M., Rommelspacher, H., Sugawa, M. D. and Dencher, N. A. (2010).Ageing alters the supramolecular architecture of OxPhos complexes in rat brain cortex Exp Gerontol 45, $563-72$.

Frick, M., Schmidt, K. and Nichols, B. J. (2007).Modulation of lateral diffusion in the plasma membrane by protein density Curr Biol 17, 462-7.

Fry, M. and Green, D. E. (1980).Cardiolipin requirement by cytochrome oxidase and the catalytic role of phospholipid Biochem Biophys Res Commun 93, 1238-46.

Gao, Y., Meyer, B., Sokolova, L., Zwicker, K., Karas, M., Brutschy, B., Peng, G. and Michel, H. (2012).Heme-copper terminal oxidase using both cytochrome $\mathrm{c}$ and ubiquinol as electron donors Proc Natl Acad Sci U S A 109, 3275-80.

Gitai, Z. (2005).The new bacterial cell biology: moving parts and subcellular architecture Cell 120, 577-86.

Gold, V. A., Robson, A., Bao, H., Romantsov, T., Duong, F. and Collinson, I. (2010).The action of cardiolipin on the bacterial translocon Proc Natl Acad Sci U S A 107, 100449.

Gomez, B., Jr. and Robinson, N. C. (1999).Phospholipase digestion of bound cardiolipin reversibly inactivates bovine cytochrome bc1 Biochemistry 38, 9031-8.

Gomez, L. A. and Hagen, T. M. (2012).Age-related decline in mitochondrial bioenergetics: Does supercomplex destabilization determine lower oxidative capacity and higher superoxide production? Semin Cell Dev Biol.

Gomez, L. A., Monette, J. S., Chavez, J. D., Maier, C. S. and Hagen, T. M. (2009).Supercomplexes of the mitochondrial electron transport chain decline in the aging rat heart Arch Biochem Biophys 490, 30-5.

Greenfield, D., McEvoy, A. L., Shroff, H., Crooks, G. E., Wingreen, N. S., Betzig, E. and Liphardt, J. (2009).Self-organization of the Escherichia coli chemotaxis network imaged with super-resolution light microscopy PLoS Biol 7, e1000137. 
Grivennikova, V. G. and Vinogradov, A. D. (2006).Generation of superoxide by the mitochondrial Complex I Biochim Biophys Acta 1757, 553-61.

Groger, T., Nathoo, S., Ku, T., Sikora, C., Turner, R. J. and Prenner, E. J. (2012).Real-time imaging of lipid domains and distinct coexisting membrane protein clusters Chem Phys Lipids 165, 216-24.

Guiral, M., Prunetti, L., Lignon, S., Lebrun, R., Moinier, D. and Giudici-Orticonit, M. T. (2009).New insights into the respiratory chains of the chemolithoautotrophic and hyperthermophilic bacterium Aquifex aeolicus J Proteome Res 8, 1717-30.

Guiral, M., Tron, P., Aubert, C., Gloter, A., Iobbi-Nivol, C. and Giudici-Orticoni, M. T. (2005).A membrane-bound multienzyme, hydrogen-oxidizing, and sulfur-reducing complex from the hyperthermophilic bacterium Aquifex aeolicus $J$ Biol Chem 280 , 42004-15.

Guo, D. and Tropp, B. E. (2000).A second Escherichia coli protein with CL synthase activity Biochim Biophys Acta 1483, 263-74.

Hackenbrock, C. R. (1968).Ultrastructural bases for metabolically linked mechanical activity in mitochondria. II. Electron transport-linked ultrastructural transformations in mitochondria J Cell Biol 37, 345-69.

Hackenbrock, C. R., Chazotte, B. and Gupte, S. S. (1986).The random collision model and a critical assessment of diffusion and collision in mitochondrial electron transport $J$ Bioenerg Biomembr 18, 331-68.

Hayer-Hartl, M., Schagger, H., von Jagow, G. and Beyer, K. (1992).Interactions of phospholipids with the mitochondrial cytochrome-c reductase studied by spin-label ESR and NMR spectroscopy Eur J Biochem 209, 423-30.

Helbig, A. O., de Groot, M. J., van Gestel, R. A., Mohammed, S., de Hulster, E. A., Luttik, M. A., Daran-Lapujade, P., Pronk, J. T., Heck, A. J. and Slijper, M. (2009).A threeway proteomics strategy allows differential analysis of yeast mitochondrial membrane protein complexes under anaerobic and aerobic conditions Proteomics 9, 4787-98.

Hiraoka, S., Matsuzaki, H. and Shibuya, I. (1993).Active increase in cardiolipin synthesis in the stationary growth phase and its physiological significance in Escherichia coli FEBS Lett 336, 221-4.

Hofmann, A. D., Beyer, M., Krause-Buchholz, U., Wobus, M., Bornhauser, M. and Rodel, G. (2012).OXPHOS supercomplexes as a hallmark of the mitochondrial phenotype of adipogenic differentiated human MSCs PLoS One 7, e35160. 
Horsefield, R., Yankovskaya, V., Sexton, G., Whittingham, W., Shiomi, K., Omura, S., Byrne, B., Cecchini, G. and Iwata, S. (2006).Structural and computational analysis of the quinone-binding site of complex II (succinate-ubiquinone oxidoreductase): a mechanism of electron transfer and proton conduction during ubiquinone reduction $J$ Biol Chem 281, 7309-16.

Huang, K. C., Mukhopadhyay, R. and Wingreen, N. S. (2006).A curvature-mediated mechanism for localization of lipids to bacterial poles PLoS Comput Biol 2, e151.

Huang, K. C. and Ramamurthi, K. S. (2010).Macromolecules that prefer their membranes curvy Mol Microbiol 76, 822-32.

Hunte, C. (2005).Specific protein-lipid interactions in membrane proteins Biochem Soc Trans 33, 938-42.

Iwasaki, T., Matsuura, K. and Oshima, T. (1995).Resolution of the aerobic respiratory system of the thermoacidophilic archaeon, Sulfolobus sp. strain 7. I. The archaeal terminal oxidase supercomplex is a functional fusion of respiratory complexes III and IV with no c-type cytochromes $J$ Biol Chem 270, 30881-92.

Jacoby, G. H. and Young, K. D. (1988).Unequal distribution of penicillin-binding proteins among inner membrane vesicles of Escherichia coli J Bacteriol 170, 3660-7.

Janzon, J., Ludwig, B. and Malatesta, F. (2007).Electron transfer kinetics of soluble fragments indicate a direct interaction between complex III and the caa3 oxidase in Thermus thermophilus IUBMB Life 59, 563-9.

Johnson, A. S., van Horck, S. and Lewis, P. J. (2004).Dynamic localization of membrane proteins in Bacillus subtilis Microbiology 150, 2815-24.

Jormakka, M., Tornroth, S., Abramson, J., Byrne, B. and Iwata, S. (2002a).Purification and crystallization of the respiratory complex formate dehydrogenase-N from Escherichia coli Acta Crystallogr D Biol Crystallogr 58, 160-2.

Jormakka, M., Tornroth, S., Byrne, B. and Iwata, S. (2002b).Molecular basis of proton motive force generation: structure of formate dehydrogenase-N Science 295, 1863-8.

Jormakka, M., Yokoyama, K., Yano, T., Tamakoshi, M., Akimoto, S., Shimamura, T., Curmi, P. and Iwata, S. (2008).Molecular mechanism of energy conservation in polysulfide respiration Nat Struct Mol Biol 15, 730-7.

Kanemasa, Y., Yoshioka, T. and Hayashi, H. (1972).Alteration of the phospholipid composition of Staphylococcus aureus cultured in medium containing $\mathrm{NaCl}$ Biochim Biophys Acta 280, 444-50. 
Kawai, F., Hara, H., Takamatsu, H., Watabe, K. and Matsumoto, K. (2006).Cardiolipin enrichment in spore membranes and its involvement in germination of Bacillus subtilis Marburg Genes Genet Syst 81, 69-76.

Kawai, F., Shoda, M., Harashima, R., Sadaie, Y., Hara, H. and Matsumoto, K. (2004).Cardiolipin domains in Bacillus subtilis marburg membranes J Bacteriol 186, 1475-83.

Keefe, R. G. and Maier, R. J. (1993).Purification and characterization of an O2-utilizing cytochrome-c oxidase complex from Bradyrhizobium japonicum bacteroid membranes Biochim Biophys Acta 1183, 91-104.

Kelly, D. J. (1998). The physiology and metabolism of the human gastric pathogen Helicobacter pylori Adv Microb Physiol 40, 137-89.

Kern, M. and Simon, J. (2009).Electron transport chains and bioenergetics of respiratory nitrogen metabolism in Wolinella succinogenes and other Epsilonproteobacteria Biochim Biophys Acta 1787, 646-56.

Kiebish, M. A., Han, X., Cheng, H., Chuang, J. H. and Seyfried, T. N. (2008).Cardiolipin and electron transport chain abnormalities in mouse brain tumor mitochondria: lipidomic evidence supporting the Warburg theory of cancer J Lipid Res 49, 2545-56.

Kikuchi, S., Shibuya, I. and Matsumoto, K. (2000).Viability of an Escherichia coli pgsA null mutant lacking detectable phosphatidylglycerol and cardiolipin J Bacteriol 182, 371-6.

Kimura, H. and Futai, M. (1978).Effects of phospholipids on L-lactate dehydrogenase from membranes of Escherichia coli. Activation and stabilization of the enzyme with phospholipids J Biol Chem 253, 1095-110.

Kirchhoff, H. (2008a).Molecular crowding and order in photosynthetic membranes Trends Plant Sci 13, 201-7.

Kirchhoff, H. (2008b).Significance of protein crowding, order and mobility for photosynthetic membrane functions Biochem Soc Trans 36, 967-70.

Kirchhoff, H., Haferkamp, S., Allen, J. F., Epstein, D. B. and Mullineaux, C. W. (2008).Protein diffusion and macromolecular crowding in thylakoid membranes Plant Physiol 146, 1571-8.

Kita, K., Konishi, K. and Anraku, Y. (1984).Terminal oxidases of Escherichia coli aerobic respiratory chain. I. Purification and properties of cytochrome b562-o complex from cells in the early exponential phase of aerobic growth $J$ Biol Chem 259, 3368-74.

Kitagawa, M., Ara, T., Arifuzzaman, M., Ioka-Nakamichi, T., Inamoto, E., Toyonaga, H. and Mori, H. (2005).Complete set of ORF clones of Escherichia coli ASKA library (a 
complete set of E. coli K-12 ORF archive): unique resources for biological research DNA Res 12, 291-9.

Kleinschroth, T., Castellani, M., Trinh, C. H., Morgner, N., Brutschy, B., Ludwig, B. and Hunte, C. (2011).X-ray structure of the dimeric cytochrome bc(1) complex from the soil bacterium Paracoccus denitrificans at 2.7-A resolution Biochim Biophys Acta 1807, 1606-15.

Koch, H. U., Haas, R. and Fischer, W. (1984). The role of lipoteichoic acid biosynthesis in membrane lipid metabolism of growing Staphylococcus aureus Eur J Biochem 138, 357-63.

Koprivnjak, T., Zhang, D., Ernst, C. M., Peschel, A., Nauseef, W. M. and Weiss, J. P. (2011).Characterization of Staphylococcus aureus cardiolipin synthases 1 and 2 and their contribution to accumulation of cardiolipin in stationary phase and within phagocytes $J$ Bacteriol 193, 4134-42.

Korshunov, S. and Imlay, J. A. (2006).Detection and quantification of superoxide formed within the periplasm of Escherichia coli J Bacteriol 188, 6326-34.

Kouril, R., Dekker, J. P. and Boekema, E. J. (2012).Supramolecular organization of photosystem II in green plants Biochim Biophys Acta 1817, 2-12.

Krause, F., Reifschneider, N. H., Vocke, D., Seelert, H., Rexroth, S. and Dencher, N. A. (2004)."Respirasome"-like supercomplexes in green leaf mitochondria of spinach $J$ Biol Chem 279, 48369-75.

Kumar, M., Mommer, M. S. and Sourjik, V. (2010).Mobility of cytoplasmic, membrane, and DNA-binding proteins in Escherichia coli Biophys J 98, 552-9.

Kusumi, A., Shirai, Y. M., Koyama-Honda, I., Suzuki, K. G. and Fujiwara, T. K. (2010).Hierarchical organization of the plasma membrane: investigations by singlemolecule tracking vs. fluorescence correlation spectroscopy FEBS Lett 584, 1814-23.

Kusumi, A., Suzuki, K. G., Kasai, R. S., Ritchie, K. and Fujiwara, T. K. (2011).Hierarchical mesoscale domain organization of the plasma membrane Trends Biochem Sci 36, 60415.

Lai, E. M., Nair, U., Phadke, N. D. and Maddock, J. R. (2004).Proteomic screening and identification of differentially distributed membrane proteins in Escherichia coli $\mathrm{Mol}$ Microbiol 52, 1029-44.

Lancaster, C. R., Kroger, A., Auer, M. and Michel, H. (1999).Structure of fumarate reductase from Wolinella succinogenes at 2.2 A resolution Nature 402, 377-85. 
Lang, T. and Rizzoli, S. O. (2010).Membrane protein clusters at nanoscale resolution: more than pretty pictures Physiology (Bethesda) 25, 116-24.

Lange, C., Nett, J. H., Trumpower, B. L. and Hunte, C. (2001).Specific roles of proteinphospholipid interactions in the yeast cytochrome bc1 complex structure Embo $J \mathbf{2 0}$, 6591-600.

Laska, S. and Kletzin, A. (2000).Improved purification of the membrane-bound hydrogenasesulfur-reductase complex from thermophilic archaea using epsilon-aminocaproic acidcontaining chromatography buffers J Chromatogr B Biomed Sci Appl 737, 151-60.

Laska, S., Lottspeich, F. and Kletzin, A. (2003).Membrane-bound hydrogenase and sulfur reductase of the hyperthermophilic and acidophilic archaeon Acidianus ambivalens Microbiology 149, 2357-71.

Lee, A. G. (2004).How lipids affect the activities of integral membrane proteins Biochim Biophys Acta 1666, 62-87.

Lee, A. G. (2011a).Biological membranes: the importance of molecular detail Trends Biochem Sci 36, 493-500.

Lee, A. G. (2011b).Lipid-protein interactions Biochem Soc Trans 39, 761-6.

Lenaz, G., Fato, R., Genova, M. L., Bergamini, C., Bianchi, C. and Biondi, A. (2006).Mitochondrial Complex I: structural and functional aspects Biochim Biophys Acta 1757, 1406-20.

Lenaz, G. and Genova, M. L. (2009).Structural and functional organization of the mitochondrial respiratory chain: a dynamic super-assembly Int J Biochem Cell Biol 41, 1750-1772.

Lenaz, G. and Genova, M. L. (2010).Structure and organization of mitochondrial respiratory complexes: a new understanding of an old subject Antioxid Redox Signal 12, 9611008 .

Lenn, T., Leake, M. C. and Mullineaux, C. W. (2008a).Are Escherichia coli OXPHOS complexes concentrated in specialized zones within the plasma membrane? Biochem Soc Trans 36, 1032-6.

Lenn, T., Leake, M. C. and Mullineaux, C. W. (2008b).Clustering and dynamics of cytochrome bd-I complexes in the Escherichia coli plasma membrane in vivo $\mathrm{Mol}$ Microbiol 70, 1397-407.

Lenne, P. F., Wawrezinieck, L., Conchonaud, F., Wurtz, O., Boned, A., Guo, X. J., Rigneault, H., He, H. T. and Marguet, D. (2006).Dynamic molecular confinement in the plasma membrane by microdomains and the cytoskeleton meshwork EMBO J 25, 3245-56. 
Li, G. and Young, K. D. (2012).Isolation and identification of new inner membraneassociated proteins that localize to cell poles in Escherichia coli Mol Microbiol 84, 276-95.

Lill, R., Dowhan, W. and Wickner, W. (1990).The ATPase activity of SecA is regulated by acidic phospholipids, SecY, and the leader and mature domains of precursor proteins Cell 60, 271-80.

Lingwood, D. and Simons, K. (2010).Lipid rafts as a membrane-organizing principle Science 327, 46-50.

Liu, X., Gong, X., Hicks, D. B., Krulwich, T. A., Yu, L. and Yu, C. A. (2007).Interaction between cytochrome caa3 and F1F0-ATP synthase of alkaliphilic Bacillus pseudofirmus OF4 is demonstrated by saturation transfer electron paramagnetic resonance and differential scanning calorimetry assays Biochemistry 46, 306-13.

Lopez, C. S., Alice, A. F., Heras, H., Rivas, E. A. and Sanchez-Rivas, C. (2006).Role of anionic phospholipids in the adaptation of Bacillus subtilis to high salinity Microbiology 152, 605-16.

Lopez, C. S., Heras, H., Ruzal, S. M., Sanchez-Rivas, C. and Rivas, E. A. (1998).Variations of the envelope composition of Bacillus subtilis during growth in hyperosmotic medium Curr Microbiol 36, 55-61.

Lopez, D. and Kolter, R. (2010).Functional microdomains in bacterial membranes Genes Dev 24, 1893-902.

Loura, L. M. and Prieto, M. (2011).FRET in Membrane Biophysics: An Overview Front Physiol 2, 82 .

Lovley, D. R., Holmes, D. E. and Nevin, K. P. (2004).Dissimilatory Fe(III) and Mn(IV) reduction Adv Microb Physiol 49, 219-86.

Macgilvray, M. E., Lapek, J. D., Friedman, A. E. and Quivey, R. G. (2012).Cardiolipin biosynthesis in Streptococcus mutans is regulated in response to external $\mathrm{pH}$ Microbiology.

Maloney, E., Lun, S., Stankowska, D., Guo, H., Rajagoapalan, M., Bishai, W. R. and Madiraju, M. V. (2011).Alterations in phospholipid catabolism in Mycobacterium tuberculosis lysX mutant Front Microbiol 2, 19.

Matsumoto, K. (2001).Dispensable nature of phosphatidylglycerol in Escherichia coli: dual roles of anionic phospholipids Mol Microbiol 39, 1427-33.

Matsumoto, K., Kusaka, J., Nishibori, A. and Hara, H. (2006).Lipid domains in bacterial membranes Mol Microbiol 61, 1110-7. 
McCrindle, S. L., Kappler, U. and McEwan, A. G. (2005).Microbial dimethylsulfoxide and trimethylamine-N-oxide respiration Adv Microb Physiol 50, 147-98.

McGarrity, J. T. and Armstrong, J. B. (1975).The effect of salt on phospholipid fatty acid composition in Escherichia coli K-12 Biochim Biophys Acta 398, 258-64.

McKenzie, M., Lazarou, M., Thorburn, D. R. and Ryan, M. T. (2006).Mitochondrial respiratory chain supercomplexes are destabilized in Barth Syndrome patients $J \mathrm{Mol}$ Biol 361, 462-9.

McMillin, J. B. and Dowhan, W. (2002).Cardiolipin and apoptosis Biochim Biophys Acta 1585, 97-107.

Megehee, J. A., Hosler, J. P. and Lundrigan, M. D. (2006).Evidence for a cytochrome bcc-aa3 interaction in the respiratory chain of Mycobacterium smegmatis Microbiology 152, 823-9.

Meilhac, N. and Destainville, N. (2011).Clusters of proteins in biomembranes: insights into the roles of interaction potential shapes and of protein diversity $J$ Phys Chem $B \mathbf{1 1 5}$, 7190-9.

Mignot, T. and Shaevitz, J. W. (2008). Active and passive mechanisms of intracellular transport and localization in bacteria Curr Opin Microbiol 11, 580-5.

Mikhaleva, N. I., Santini, C. L., Giordano, G., Nesmeyanova, M. A. and Wu, L. F. (1999).Requirement for phospholipids of the translocation of the trimethylamine Noxide reductase through the Tat pathway in Escherichia coli FEBS Lett 463, 331-5.

Mileykovskaya, E. and Dowhan, W. (2000).Visualization of phospholipid domains in Escherichia coli by using the cardiolipin-specific fluorescent dye 10-N-nonyl acridine orange $J$ Bacteriol 182, 1172-5.

Mileykovskaya, E. and Dowhan, W. (2009).Cardiolipin membrane domains in prokaryotes and eukaryotes Biochim Biophys Acta.

Mileykovskaya, E., Ryan, A. C., Mo, X., Lin, C. C., Khalaf, K. I., Dowhan, W. and Garrett, T. A. (2009).Phosphatidic acid and N-acylphosphatidylethanolamine form membrane domains in Escherichia coli mutant lacking cardiolipin and phosphatidylglycerol $J$ Biol Chem 284, 2990-3000.

Mileykovskaya, E., Sun, Q., Margolin, W. and Dowhan, W. (1998).Localization and function of early cell division proteins in filamentous Escherichia coli cells lacking phosphatidylethanolamine J Bacteriol 180, 4252-7.

Mileykovskaya, E., Zhang, M. and Dowhan, W. (2005).Cardiolipin in energy transducing membranes Biochemistry (Mosc) 70, 154-8. 
Mileykovskaya, E. I. and Dowhan, W. (1993).Alterations in the electron transfer chain in mutant strains of Escherichia coli lacking phosphatidylethanolamine $J$ Biol Chem 268, 24824-31.

Miller, K. J. (1986).Effects of monovalent and divalent salts on the phospholipid and fatty acid compositions of a halotolerant Planococcus sp Appl Environ Microbiol 52, 580-2.

Minauro-Sanmiguel, F., Wilkens, S. and Garcia, J. J. (2005).Structure of dimeric mitochondrial ATP synthase: novel F0 bridging features and the structural basis of mitochondrial cristae biogenesis Proc Natl Acad Sci U S A 102, 12356-8.

Miyazaki, C., Kuroda, M., Ohta, A. and Shibuya, I. (1985).Genetic manipulation of membrane phospholipid composition in Escherichia coli: pgsA mutants defective in phosphatidylglycerol synthesis Proc Natl Acad Sci U S A 82, 7530-4.

Moreno-Lastres, D., Fontanesi, F., Garcia-Consuegra, I., Martin, M. A., Arenas, J., Barrientos, A. and Ugalde, C. (2012).Mitochondrial complex I plays an essential role in human respirasome assembly Cell Metab 15, 324-35.

Morozova, D., Guigas, G. and Weiss, M. (2011).Dynamic structure formation of peripheral membrane proteins PLoS Comput Biol 7, e1002067.

Muchova, K., Wilkinson, A. J. and Barak, I. (2011).Changes of lipid domains in Bacillus subtilis cells with disrupted cell wall peptidoglycan FEMS Microbiol Lett 325, 92-8.

Mukhopadhyay, R., Huang, K. C. and Wingreen, N. S. (2008).Lipid localization in bacterial cells through curvature-mediated microphase separation Biophys $J$ 95, 1034-49.

Muller, F., Crofts, A. R. and Kramer, D. M. (2002).Multiple Q-cycle bypass reactions at the Qo site of the cytochrome bc1 complex Biochemistry 41, 7866-74.

Muller, F. L., Liu, Y., Abdul-Ghani, M. A., Lustgarten, M. S., Bhattacharya, A., Jang, Y. C. and Van Remmen, H. (2008).High rates of superoxide production in skeletal-muscle mitochondria respiring on both complex I- and complex II-linked substrates Biochem J 409, 491-9.

Müller, M., Mentel, M., Van Hellemond, J. J., Henze, K., Woehle, C., Gould, S. B., Yu, R., van der Giezn, M., Tielens, A. G. and Martin, W. F. (2012).Biochemistry and evolution of anaerobic energy metabolism in eukaryotes Microbiology and Molecular Biology Reviews 76, 444-495.

Nevo-Dinur, K., Govindarajan, S. and Amster-Choder, O. (2012).Subcellular localization of RNA and proteins in prokaryotes Trends Genet.

Niebisch, A. and Bott, M. (2003).Purification of a cytochrome bc-aa3 supercomplex with quinol oxidase activity from Corynebacterium glutamicum. Identification of a fourth 
subunity of cytochrome aa3 oxidase and mutational analysis of diheme cytochrome $\mathrm{c} 1$ J Biol Chem 278, 4339-46.

Niemela, P. S., Miettinen, M. S., Monticelli, L., Hammaren, H., Bjelkmar, P., Murtola, T., Lindahl, E. and Vattulainen, I. (2010).Membrane proteins diffuse as dynamic complexes with lipids J Am Chem Soc 132, 7574-5.

Nishibori, A., Kusaka, J., Hara, H., Umeda, M. and Matsumoto, K. (2005).Phosphatidylethanolamine domains and localization of phospholipid synthases in Bacillus subtilis membranes $J$ Bacteriol 187, 2163-74.

Nishijima, S., Asami, Y., Uetake, N., Yamagoe, S., Ohta, A. and Shibuya, I. (1988).Disruption of the Escherichia coli cls gene responsible for cardiolipin synthesis J Bacteriol 170, 775-80.

Nury, H., Dahout-Gonzalez, C., Trezeguet, V., Lauquin, G., Brandolin, G. and PebayPeyroula, E. (2005).Structural basis for lipid-mediated interactions between mitochondrial ADP/ATP carrier monomers FEBS Lett 579, 6031-6.

Palmer, T., Sargent, F. and Berks, B. C. (2005).Export of complex cofactor-containing proteins by the bacterial Tat pathway Trends Microbiol 13, 175-80.

Palsdottir, H., Lojero, C. G., Trumpower, B. L. and Hunte, C. (2003).Structure of the yeast cytochrome bc1 complex with a hydroxyquinone anion Qo site inhibitor bound $J$ Biol Chem 278, 31303-11.

Paradies, G., Petrosillo, G., Paradies, V. and Ruggiero, F. M. (2011).Mitochondrial dysfunction in brain aging: role of oxidative stress and cardiolipin Neurochem Int 58, 447-57.

Paradies, G., Petrosillo, G., Pistolese, M., Di Venosa, N., Federici, A. and Ruggiero, F. M. (2004).Decrease in mitochondrial complex I activity in ischemic/reperfused rat heart: involvement of reactive oxygen species and cardiolipin Circ Res 94, 53-9.

Paumard, P., Vaillier, J., Coulary, B., Schaeffer, J., Soubannier, V., Mueller, D. M., Brethes, D., di Rago, J. P. and Velours, J. (2002).The ATP synthase is involved in generating mitochondrial cristae morphology Embo J 21, 221-30.

Pereira, M. M., Carita, J. N. and Teixeira, M. (1999a).Membrane-bound electron transfer chain of the thermohalophilic bacterium Rhodothermus marinus: a novel multihemic cytochrome bc, a new complex III Biochemistry 38, 1268-75.

Pereira, M. M., Carita, J. N. and Teixeira, M. (1999b).Membrane-bound electron transfer chain of the thermohalophilic bacterium Rhodothermus marinus: characterization of the iron-sulfur centers from the dehydrogenases and investigation of the high-potential 
iron-sulfur protein function by in vitro reconstitution of the respiratory chain Biochemistry 38, 1276-83.

Pessi, G., Ahrens, C. H., Rehrauer, H., Lindemann, A., Hauser, F., Fischer, H. M. and Hennecke, H. (2007).Genome-wide transcript analysis of Bradyrhizobium japonicum bacteroids in soybean root nodules Mol Plant Microbe Interact 20, 1353-63.

Petrosillo, G., Matera, M., Casanova, G., Ruggiero, F. M. and Paradies, G. (2008).Mitochondrial dysfunction in rat brain with aging Involvement of complex I, reactive oxygen species and cardiolipin Neurochem Int 53, 126-31.

Petrosillo, G., Matera, M., Moro, N., Ruggiero, F. M. and Paradies, G. (2009).Mitochondrial complex I dysfunction in rat heart with aging: critical role of reactive oxygen species and cardiolipin Free Radic Biol Med 46, 88-94.

Petrosillo, G., Ruggiero, F. M., Di Venosa, N. and Paradies, G. (2003a).Decreased complex III activity in mitochondria isolated from rat heart subjected to ischemia and reperfusion: role of reactive oxygen species and cardiolipin FASEB J 17, 714-6.

Petrosillo, G., Ruggiero, F. M. and Paradies, G. (2003b).Role of reactive oxygen species and cardiolipin in the release of cytochrome c from mitochondria FASEB J 17, 2202-8.

Pfeiffer, K., Gohil, V., Stuart, R. A., Hunte, C., Brandt, U., Greenberg, M. L. and Schagger, H. (2003).Cardiolipin stabilizes respiratory chain supercomplexes J Biol Chem 278, 52873-80.

Phillips, R., Ursell, T., Wiggins, P. and Sens, P. (2009).Emerging roles for lipids in shaping membrane-protein function Nature 459, 379-85.

Poole, R. K. and Cook, G. M. (2000).Redundancy of aerobic respiratory chains in bacteria? Routes, reasons and regulation Adv Microb Physiol 43, 165-224.

Poore, V. M. and Ragan, C. I. (1982).A spin label study of the lipid boundary layer of mitochondrial NADH-ubiquinone oxidoreductase Biochim Biophys Acta 693, 105-12.

Poveda, J. A., Fernandez, A. M., Encinar, J. A. and Gonzalez-Ros, J. M. (2008).Proteinpromoted membrane domains Biochim Biophys Acta 1778, 1583-90.

Prunetti, L., Infossi, P., Brugna, M., Ebel, C., Giudici-Orticoni, M. T. and Guiral, M. (2010).New functional sulfide oxidase-oxygen reductase supercomplex in the membrane of the hyperthermophilic bacterium Aquifex aeolicus $J$ Biol Chem 285, 41815-26.

Qin, L., Hiser, C., Mulichak, A., Garavito, R. M. and Ferguson-Miller, S. (2006).Identification of conserved lipid/detergent-binding sites in a high-resolution 
structure of the membrane protein cytochrome c oxidase Proc Natl Acad Sci U S A 103, 16117-22.

Quinlan, C. L., Orr, A. L., Perevoshchikova, I. V., Treberg, J. R., Ackrell, B. A. and Brand, M. D. (2012).Mitochondrial complex II can generate reactive oxygen species at high rates in both the forward and reverse reactions. $J$ Biol Chem in press, DOI: 10.1074/jbc.M112.374629.

Ramadurai, S., Holt, A., Krasnikov, V., van den Bogaart, G., Killian, J. A. and Poolman, B. (2009).Lateral diffusion of membrane proteins J Am Chem Soc 131, 12650-6.

Ramamurthi, K. S. (2010).Protein localization by recognition of membrane curvature Curr Opin Microbiol 13, 753-7.

Ramirez-Aguilar, S. J., Keuthe, M., Rocha, M., Fedyaev, V. V., Kramp, K., Gupta, K. J., Rasmusson, A. G., Schulze, W. X. and van Dongen, J. T. (2011).The composition of plant mitochondrial supercomplexes changes with oxygen availability $J$ Biol Chem 286, 43045-53.

Randle, C. L., Albro, P. W. and Dittmer, J. C. (1969).The phosphoglyceride composition of Gram-negative bacteria and the changes in composition during growth Biochim Biophys Acta 187, 214-20.

Reddy, T. L. and Weber, M. M. (1986).Solubilization, purification, and characterization of succinate dehydrogenase from membranes of Mycobacterium phlei J Bacteriol 167, 16.

Refojo, P. N., Sousa, F. L., Teixeira, M. and Pereira, M. M. (2010a).The alternative complex III: a different architecture using known building modules Biochim Biophys Acta 1797, 1869-76.

Refojo, P. N., Teixeira, M. and Pereira, M. M. (2010b).The alternative complex III of Rhodothermus marinus and its structural and functional association with caa3 oxygen reductase Biochim Biophys Acta 1797, 1477-82.

Richardson, D. J. (2000).Bacterial respiration: a flexible process for a changing environment Microbiology 146 ( Pt 3), 551-71.

Robinson, N. C. (1993).Functional binding of cardiolipin to cytochrome c oxidase $J$ Bioenerg Biomembr 25, 153-63.

Robinson, N. C., Zborowski, J. and Talbert, L. H. (1990).Cardiolipin-depleted bovine heart cytochrome c oxidase: binding stoichiometry and affinity for cardiolipin derivatives Biochemistry 29, 8962-9. 
Romantsov, T., Battle, A. R., Hendel, J. L., Martinac, B. and Wood, J. M. (2010).Protein localization in Escherichia coli cells: comparison of the cytoplasmic membrane proteins ProP, LacY, ProW, AqpZ, MscS, and MscL J Bacteriol 192, 912-24.

Romantsov, T., Guan, Z. and Wood, J. M. (2009).Cardiolipin and the osmotic stress responses of bacteria Biochim Biophys Acta.

Rosca, M. G. and Hoppel, C. L. (2009).New aspects of impaired mitochondrial function in heart failure J Bioenerg Biomembr 41, 107-12.

Rudner, D. Z. and Losick, R. (2010).Protein subcellular localization in bacteria Cold Spring Harb Perspect Biol 2, a000307.

Ruprecht, J., Iwata, S., Rothery, R. A., Weiner, J. H., Maklashina, E. and Cecchini, G. (2011).Perturbation of the quinone-binding site of complex II alters the electronic properties of the proximal [3Fe-4S] iron-sulfur cluster J Biol Chem 286, 12756-65.

Ruprecht, J., Yankovskaya, V., Maklashina, E., Iwata, S. and Cecchini, G. (2009).Structure of Escherichia coli succinate:quinone oxidoreductase with an occupied and empty quinone-binding site $J$ Biol Chem 284, 29836-46.

Ryan, T. A., Myers, J., Holowka, D., Baird, B. and Webb, W. W. (1988).Molecular crowding on the cell surface Science 239, 61-4.

Sakamoto, J., Shibata, T., Mine, T., Miyahara, R., Torigoe, T., Noguchi, S., Matsushita, K. and Sone, N. (2001).Cytochrome c oxidase contains an extra charged amino acid cluster in a new type of respiratory chain in the amino-acid-producing Gram-positive bacterium Corynebacterium glutamicum Microbiology 147, 2865-71.

Schagger, H., de Coo, R., Bauer, M. F., Hofmann, S., Godinot, C. and Brandt, U. (2004).Significance of respirasomes for the assembly/stability of human respiratory chain complex I J Biol Chem 279, 36349-53.

Schagger, H., Hagen, T., Roth, B., Brandt, U., Link, T. A. and von Jagow, G. (1990).Phospholipid specificity of bovine heart bc1 complex Eur J Biochem 190, 123 30 .

Schagger, H. and Pfeiffer, K. (2000).Supercomplexes in the respiratory chains of yeast and mammalian mitochondria Embo J 19, 1777-83.

Schlame, M. and Ren, M. (2006).Barth syndrome, a human disorder of cardiolipin metabolism FEBS Lett 580, 5450-5.

Schlame, M. and Ren, M. (2009).The role of cardiolipin in the structural organization of mitochondrial membranes Biochim Biophys Acta 1788, 2080-3. 
Schneider, D., Pohl, T., Walter, J., Dorner, K., Kohlstadt, M., Berger, A., Spehr, V. and Friedrich, T. (2008).Assembly of the Escherichia coli NADH:ubiquinone oxidoreductase (complex I) Biochim Biophys Acta 1777, 735-9.

Schwall, C. T., Greenwood, V. L. and Alder, N. N. (2012). The Stability and Activity of Respiratory Complex II is Cardiolipin-Dependent Biochim Biophys Acta.

Sedlak, E. and Robinson, N. C. (1999).Phospholipase A(2) digestion of cardiolipin bound to bovine cytochrome $\mathrm{c}$ oxidase alters both activity and quaternary structure Biochemistry 38, 14966-72.

Shapiro, L., McAdams, H. and Losick, R. (2009). Why and how bacteria localize proteins Science 326, 11225-8.

Shibuya, I. (1992).Metabolic regulations and biological functions of phospholipids in Escherichia coli Prog Lipid Res 31, 245-99.

Shibuya, I., Miyazaki, C. and Ohta, A. (1985).Alteration of phospholipid composition by combined defects in phosphatidylserine and cardiolipin synthases and physiological consequences in Escherichia coli J Bacteriol 161, 1086-92.

Shinzawa-Itoh, K., Aoyama, H., Muramoto, K., Terada, H., Kurauchi, T., Tadehara, Y., Yamasaki, A., Sugimura, T., Kurono, S., Tsujimoto, K., Mizushima, T., Yamashita, E., Tsukihara, T. and Yoshikawa, S. (2007).Structures and physiological roles of 13 integral lipids of bovine heart cytochrome c oxidase Embo J 26, 1713-25.

Short, S. A. and White, D. C. (1971).Metabolism of phosphatidylglycerol, lysylphosphatidylglycerol, and cardiolipin of Staphylococcus aureus $J$ Bacteriol 108, 219-26.

Simon, J., Kern, M., Hermann, B., Einsle, O. and Butt, J. N. (2011).Physiological function and catalytic versatility of bacterial multihaem cytochromes $\mathrm{c}$ involved in nitrogen and sulfur cycling Biochem Soc Trans 39, 1864-70.

Simon, J., van Spanning, R. J. and Richardson, D. J. (2008).The organisation of proton motive and non-proton motive redox loops in prokaryotic respiratory systems Biochim Biophys Acta 1777, 1480-90.

Singer, S. J. and Nicolson, G. L. (1972).The fluid mosaic model of the structure of cell membranes Science 175, 720-31.

Smith, A. W. (2012).Lipid-protein interactions in biological membranes: a dynamic perspective Biochim Biophys Acta 1818, 172-7. 
Sone, N., Sekimachi, M. and Kutoh, E. (1987).Identification and properties of a quinol oxidase super-complex composed of a bc1 complex and cytochrome oxidase in the thermophilic bacterium PS3 J Biol Chem 262, 15386-91.

Soubannier, V., Vaillier, J., Paumard, P., Coulary, B., Schaeffer, J. and Velours, J. (2002).In the absence of the first membrane-spanning segment of subunit 4(b), the yeast ATP synthase is functional but does not dimerize or oligomerize $J$ Biol Chem 277, 1073945.

Sousa, P. M., Silva, S. T., Hood, B. L., Charro, N., Carita, J. N., Vaz, F., Penque, D., Conrads, T. P. and Melo, A. M. (2011).Supramolecular organizations in the aerobic respiratory chain of Escherichia coli Biochimie 93, 418-25.

Stolz, J. F., Basu, P., Santini, J. M. and Oremland, R. S. (2006).Arsenic and selenium in microbial metabolism Annu Rev Microbiol 60, 107-30.

Strauss, M., Hofhaus, G., Schroder, R. R. and Kuhlbrandt, W. (2008).Dimer ribbons of ATP synthase shape the inner mitochondrial membrane Embo J 27, 1154-60.

Strogolova, V., Furness, A., Robb-McGrath, M., Garlich, J. and Stuart, R. A. (2012).Rcf1 and Rcf2, members of the hypoxia-induced gene 1 protein family, are critical components of the mitochondrial cytochrome bc1-cytochrome c oxidase supercomplex Mol Cell Biol 32, 1363-73.

Stroh, A., Anderka, O., Pfeiffer, K., Yagi, T., Finel, M., Ludwig, B. and Schagger, H. (2004).Assembly of respiratory complexes I, III, and IV into NADH oxidase supercomplex stabilizes complex I in Paracoccus denitrificans J Biol Chem 279, 50007.

Stuart, R. A. (2008).Supercomplex organization of the oxidative phosphorylation enzymes in yeast mitochondria $J$ Bioenerg Biomembr 40, 411-7.

Sturgis, J. N. and Niederman, R. A. (2008).Atomic force microscopy reveals multiple patterns of antenna organization in purple bacteria: implications for energy transduction mechanisms and membrane modeling Photosynth Res 95, 269-78.

Suzuki, H., Nishiyama, K. and Tokuda, H. (1999).Increases in acidic phospholipid contents specifically restore protein translocation in a cold-sensitive secA or secG null mutant $J$ Biol Chem 274, 31020-4.

Tanaka, T., Inoue, M., Sakamoto, J. and Sone, N. (1996).Intra- and inter-complex crosslinking of subunits in the quinol oxidase super-complex from thermophilic Bacillus PS3 J Biochem 119, 482-6. 
Tanaka, Y., Anraku, Y. and Futai, M. (1976).Escherichia coli membrane D-lactate dehydrogenase. Isolation of the enzyme in aggregated from and its activation by Triton X-100 and phospholipids J Biochem 80, 821-30.

Thomson, J. W. and Shapiro, B. M. (1981). The respiratory chain NADH dehydrogenase of Escherichia coli. Isolation of an NADH:quinone oxidoreductase from membranes and comparison with the membrane-bound NADH:dichlorophenolindophenol oxidoreductase $J$ Biol Chem 256, 3077-84.

Tielens, A. G., Rotte, C., van Hellemond, J. J. and Martin, W. (2002).Mitochondria as we don't know them Trends Biochem Sci 27, 564-72.

Treberg, J. R., Quinlan, C. L. and Brand, M. D. (2011).Evidence for two sites of superoxide production by mitochondrial NADH-ubiquinone oxidoreductase (complex I) $\mathrm{J}$ Biol Chem 286, 27103-10.

Trouillard, M., Meunier, B. and Rappaport, F. (2011).Questioning the functional relevance of mitochondrial supercomplexes by time-resolved analysis of the respiratory chain Proc Natl Acad Sci U S A 108, E1027-34.

van de Pas, B. A., Smidt, H., Hagen, W. R., van der Oost, J., Schraa, G., Stams, A. J. and de Vos, W. M. (1999).Purification and molecular characterization of ortho-chlorophenol reductive dehalogenase, a key enzyme of halorespiration in Desulfitobacterium dehalogenans $J$ Biol Chem 274, 20287-92.

Vanounou, S., Parola, A. H. and Fishov, I. (2003).Phosphatidylethanolamine and phosphatidylglycerol are segregated into different domains in bacterial membrane. A study with pyrene-labelled phospholipids Mol Microbiol 49, 1067-79.

Ventrella, A., Catucci, L., Mascolo, G., Corcelli, A. and Agostiano, A. (2007).Isolation and characterization of lipids strictly associated to PSII complexes: focus on cardiolipin structural and functional role Biochim Biophys Acta 1768, 1620-7.

Vonck, J. and Schafer, E. (2009).Supramolecular organization of protein complexes in the mitochondrial inner membrane Biochim Biophys Acta 1793, 117-24.

Vukotic, M., Oeljeklaus, S., Wiese, S., Vogtle, F. N., Meisinger, C., Meyer, H. E., Zieseniss, A., Katschinski, D. M., Jans, D. C., Jakobs, S., Warscheid, B., Rehling, P. and Deckers, M. (2012).Rcf1 mediates cytochrome oxidase assembly and respirasome formation, revealing heterogeneity of the enzyme complex Cell Metab 15, 336-47.

Wang, J., Cao, Y., Chen, Y., Gardner, P. and Steiner, D. F. (2006).Pancreatic beta cells lack a low glucose and $\mathrm{O} 2$-inducible mitochondrial protein that augments cell survival Proc Natl Acad Sci U S A 103, 10636-41. 
Wittig, I. and Schagger, H. (2009).Supramolecular organization of ATP synthase and respiratory chain in mitochondrial membranes Biochim Biophys Acta 1787, 672-80.

Woronowicz, K., Sha, D., Frese, R. N., Sturgis, J. N., Nanda, V. and Niederman, R. A. (2011). The effects of protein crowding in bacterial photosynthetic membranes on the flow of quinone redox species between the photochemical reaction center and the ubiquinol-cytochrome c2 oxidoreductase Metallomics 3, 765-74.

Xu, Y., Malhotra, A., Ren, M. and Schlame, M. (2006).The enzymatic function of tafazzin $J$ Biol Chem 281, 39217-24.

Yankovskaya, V., Horsefield, R., Tornroth, S., Luna-Chavez, C., Miyoshi, H., Leger, C., Byrne, B., Cecchini, G. and Iwata, S. (2003).Architecture of succinate dehydrogenase and reactive oxygen species generation Science 299, 700-4.

$\mathrm{Yu}, \mathrm{C}$. A. and $\mathrm{Yu}, \mathrm{L}$. (1980). Structural role of phospholipids in ubiquinol-cytochrome c reductase Biochemistry 19, 5715-20.

Zhang, M., Mileykovskaya, E. and Dowhan, W. (2002).Gluing the respiratory chain together. Cardiolipin is required for supercomplex formation in the inner mitochondrial membrane J Biol Chem 277, 43553-6.

Zhang, M., Mileykovskaya, E. and Dowhan, W. (2005).Cardiolipin is essential for organization of complexes III and IV into a supercomplex in intact yeast mitochondria J Biol Chem 280, 29403-8.

Zhuang, K., Vemuri, G. N. and Mahadevan, R. (2011).Economics of membrane occupancy and respiro-fermentation Mol Syst Biol 7, 500.

Zumft, W. G. and Kroneck, P. M. (2007).Respiratory transformation of nitrous oxide (N2O) to dinitrogen by Bacteria and Archaea Adv Microb Physiol 52, 107-227. 
Table 1: Supramolecular organization of Prokaryotic Respiratory systems

Aerobic and anaerobic respiratory supercomplexes that have been identified are separately listed. The enzymatic composition and overall activity of each supercomplex are indicated as well as in which microorganism it has been isolated. Small italic letters refer to the cytochromes, CI for the NADH dehydrogenase, ACIII for alternative complex III, SQR for sulfide quinone reductase, HYD for hydrogenase, SR for sulfur reductase. OR refers to oxidoreductase activity of the different supercomplexes.

Figure 1: Various models of organization of the mitochondrial respiratory chain.

The mitochondrial respiratory system is composed of 5 membrane-embedded complexes and 2 soluble electron carriers, the liposoluble ubiquinone/ubiquinols (UQ/ $\mathrm{UQH}_{2}$ ) and the hydrosoluble cytochrome c (c). Complex I oxidizes NADH and reduces UQ into $\mathrm{UQH}_{2}$. Complex II oxidizes succinate into fumarate and reduces UQ into $\mathrm{UQH}_{2}$. Complex III oxidizes $\mathrm{UQH}_{2}$ and reduces the cytochrome $c$ which ensures electron transfer to the complex IV ultimately reducing $\mathrm{O}_{2}$ into $\mathrm{H}_{2} \mathrm{O}$. Complexes I, III and IV translocate protons from the matrix ( $\mathrm{N}$ for negative side of the coupling membrane) to the intermembrane space ( $\mathrm{P}$ for positive side of the coupling membrane) and allow the establishment of a transmembrane proton gradient. The complex V phosphorylates ADP into ATP using the energy from the proton gradient. The electron transfer/transport and the proton translocation are depicted by black arrows. In $\mathbf{A}$ is shown the "liquid model" in which all components of the respiratory chain are dispersed in the inner membrane. In B is shown the "solid-state" model in which respiratory complexes are organized into supercomplexes of various composition channeling electrons to $\mathrm{O}_{2}$ and ensuring confinement of mobile electron carriers. In $\mathbf{C}$ is shown the "compartmented model", in which specialization of the inner membrane in cristae defines confined and separated spaces for proton sources (ie respiratory complexes in the form of supercomplexes) and for proton sink (ie the ATP synthase).

Figure 2: Diversity of the Escherichia coli respiratory systems

The respiratory chains in Escherichia coli are organized into three modules: primary dehydrogenases (depicted in light grey), $\mathrm{Q} / \mathrm{QH}_{2}$ and terminal reductases (depicted in dark grey). There are 15 different primary dehydrogenases, 3 types of quinones and 11 terminal reductases. $\mathrm{UQ} / \mathrm{UQH}_{2}$ stands for ubiquinone/quinol, $\mathrm{MQ} / \mathrm{MQH}_{2}$ for menaquinone/quinol and $\mathrm{DMQ} / \mathrm{DMQH}_{2}$ for demethylmenaquinone/quinol. Different redox potentials are associated with the different types of quinones. In addition to different expression patterns, any 
association of a primary dehydrogenase with a terminal reductase will not have the same consequence in terms of energy yield as expressed by $\mathrm{H}^{+} / \mathrm{e}^{-}$ratio. The activity of each respiratory enzyme is depicted, as well as the name of each structural subunit.

Figure 3: Schematic representation of the supramolecular organization of respiratory chains in rod-shaped gram-negative bacteria.

Lipids, proteins and subcellular localization are determinants for supramolecular organization of the respiratory chains in prokaryotes (see section 3). Rather than describing distinct models depicting various respiratory complexes association and the associated cues, we consider that a number of situations may coexist within the same cell (herein the gram-negative E. coli cell taken as example) depending on the physiological conditions. Primary dehydrogenases and terminal reductases (depicted as circles) evenly distributed or not in the inner membrane of the rod-shaped bacteria can further oligomerized. Cardiolipin microdomains of the inner membrane are present at the cell poles and represented as a continuous black line. The outer membrane is pictured in dark grey while the peptidoglycan is depicted by a thin black line between both membranes. Cardiolipin may contribute to polar localization of a subset of respiratory complexes. Proteic factors other than the respiratory complexes themselves may participate to supercomplex formation and even to their uneven distribution. 
A

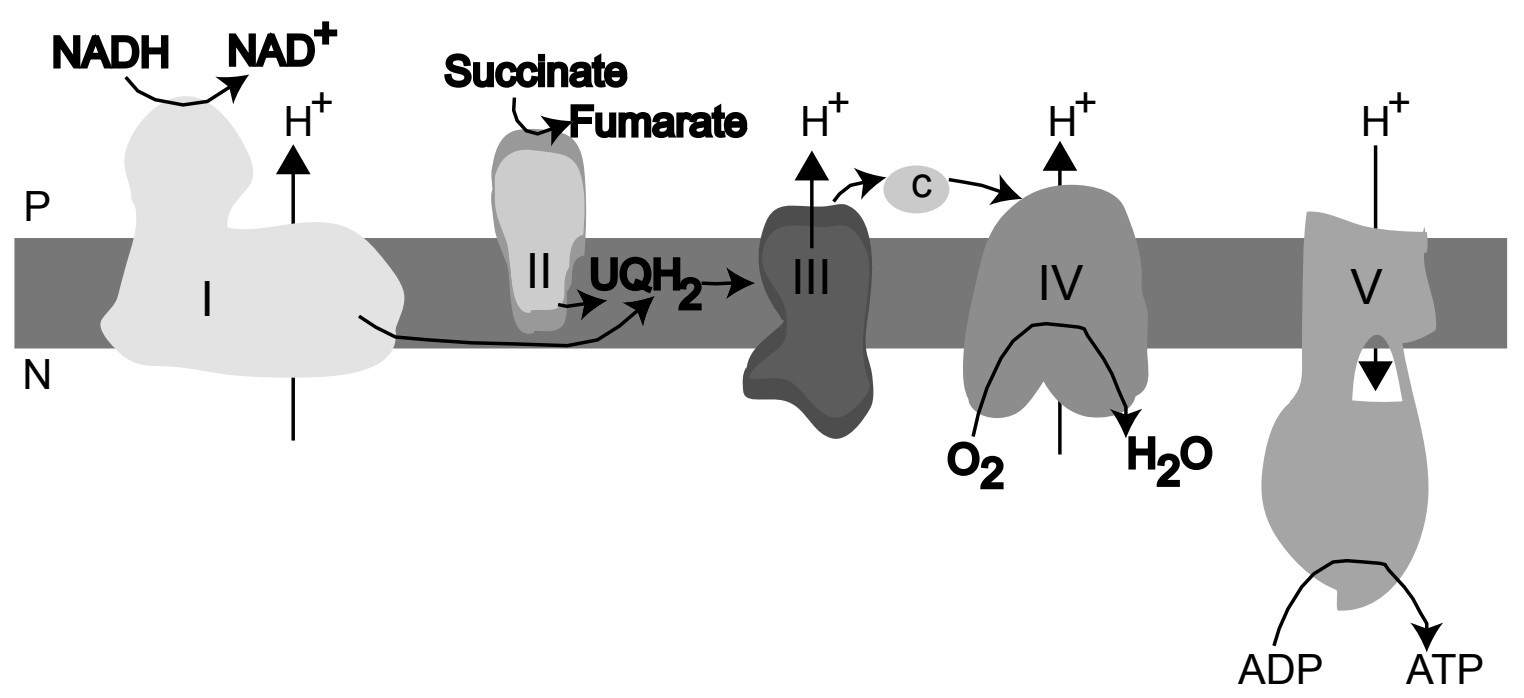

B

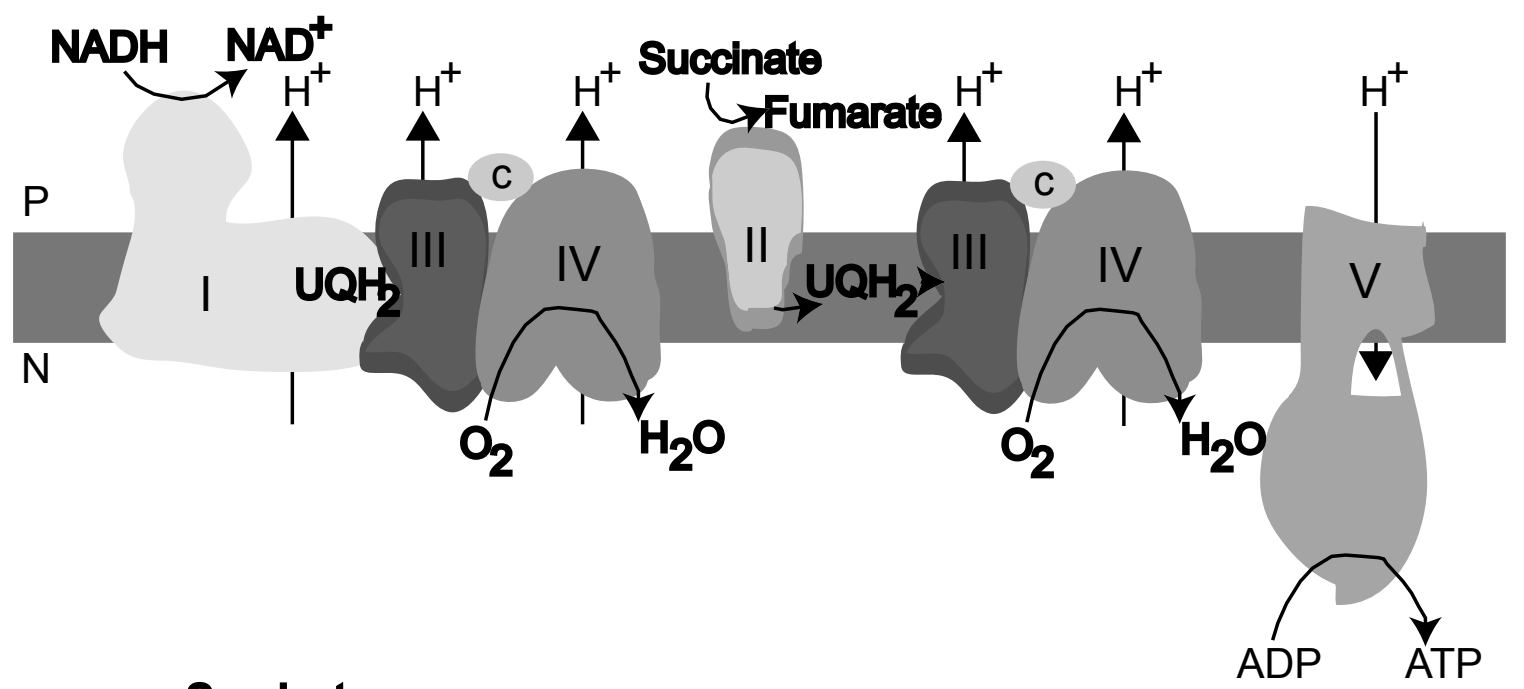

C

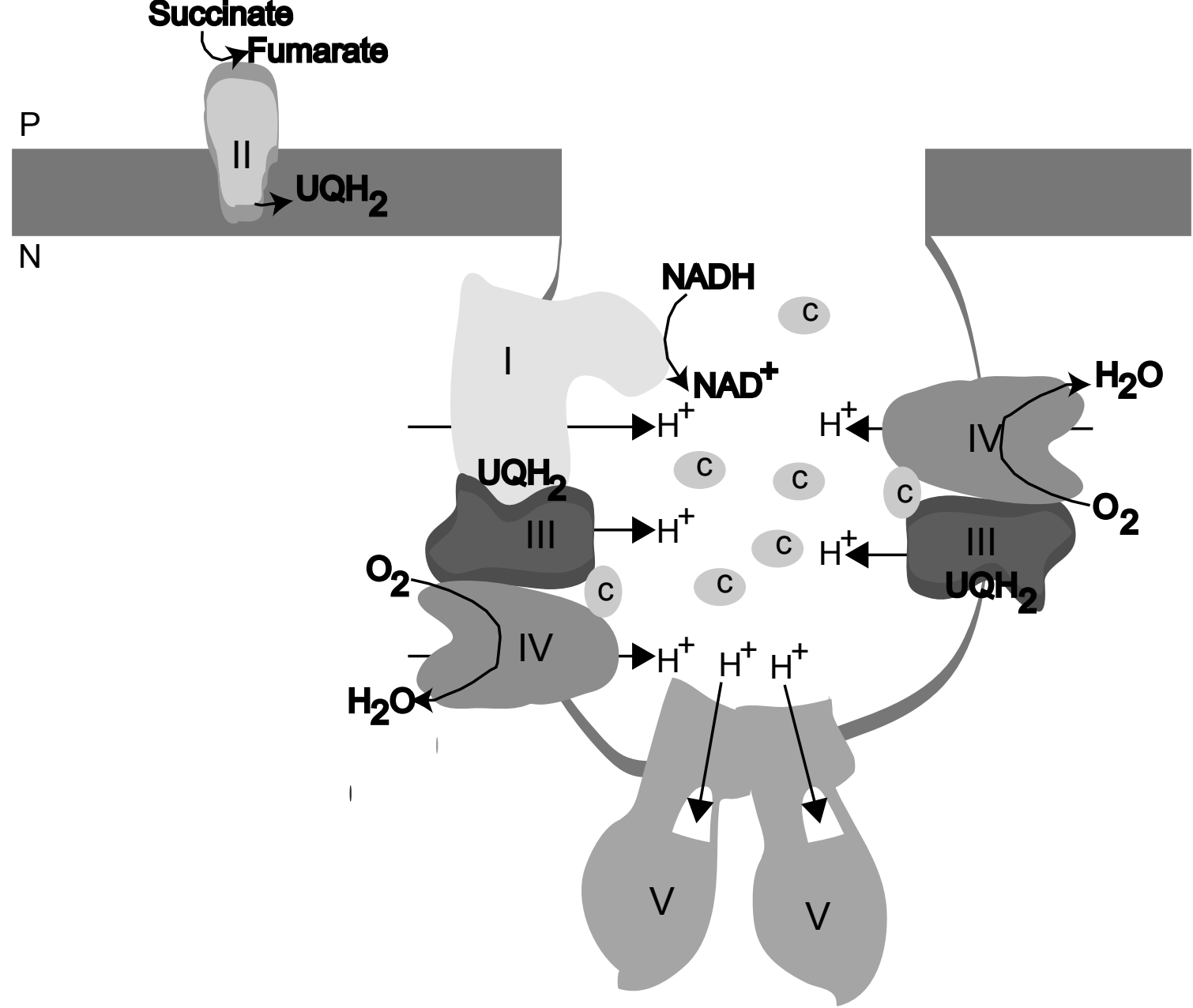




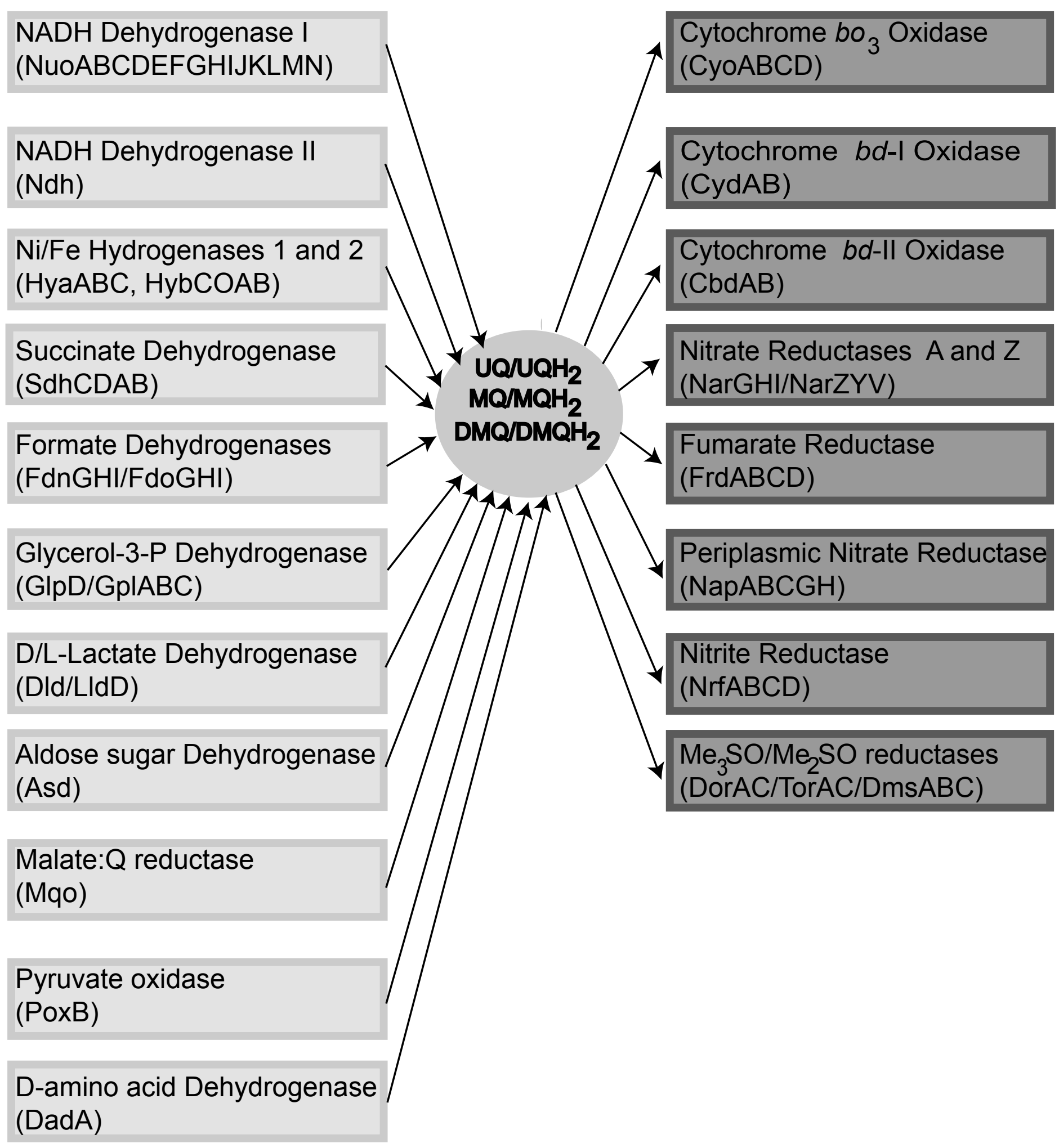




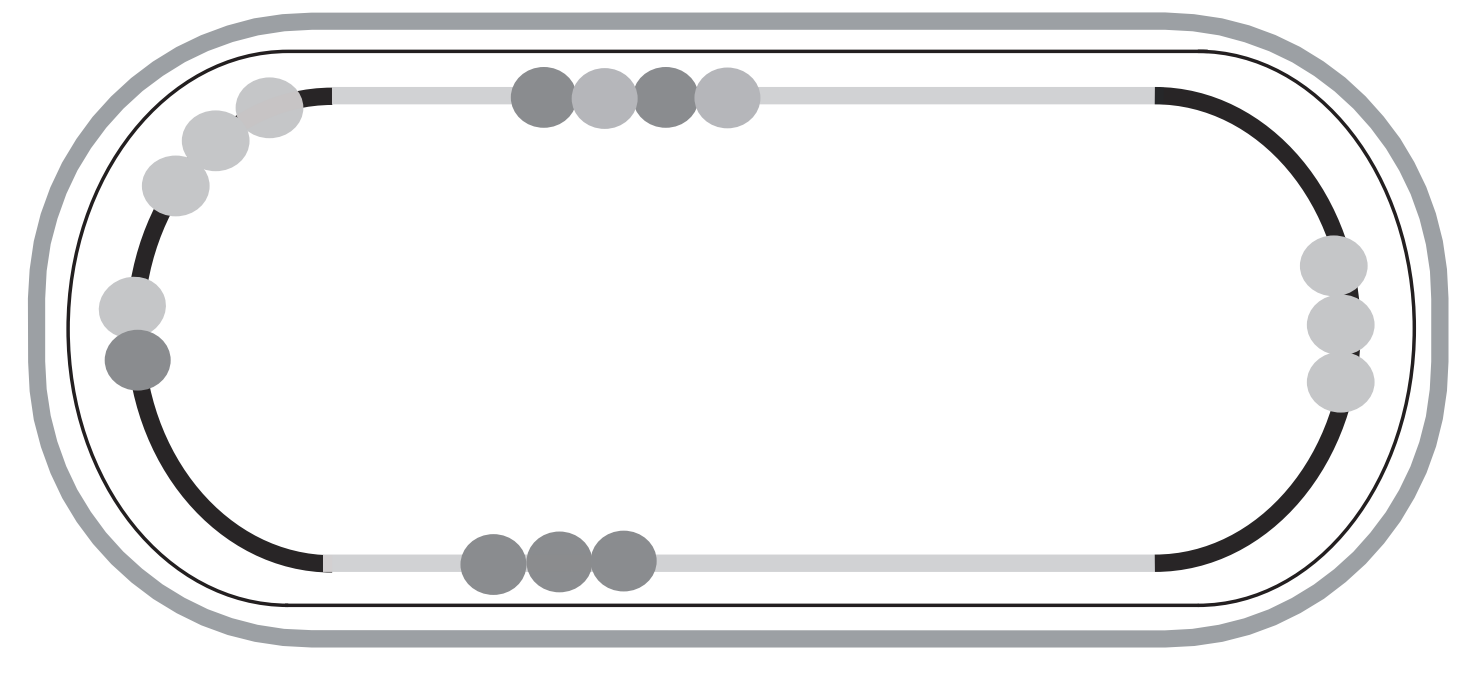


Supramolecular organization of prokaryotic respiratory systems

\section{Aerobic respiratory Activity Microorganisms} supercomplexes
$b c_{1}-a a_{3}$
Quinol:: $\mathrm{O}_{2} \mathrm{OR}$
P. denitrificans
Bacterium PS3
(Berry and Trumpower 1985)
A. aeolicus
Sulfolobus sp. Strain 7
(Sone et al., 1987)
(Guiral et al., 2009)
(Iwasaki et al., 1995)
bcc-aa 3
M. smegmatis
C. glutanicum
(Megehee et al., 2006)
(Niebisch and Bott, 2003)
$b c_{1}-b a_{3}$
A. aeolicus
(Gao et al., 2012)
$b c_{1}-c b b_{3}$
B. japonicum
(Keefe and Maier, 1993)
$\mathrm{ACIII-} c b b_{3}$
R. marinus
(Rejofo et al., 2010)

$\mathrm{Cl}-b c_{1}-\mathrm{aa}_{3} \quad \mathrm{NADH}:: \mathrm{O}_{2}$ OR P. denitrificans

(Stroh et al., 2004)

SQR- $b c_{1}-b a_{3}$

$\mathrm{H}_{2} \mathrm{~S}:: \mathrm{O}_{2} \mathrm{OR}$

A. aeolicus

(Prunetti et al., 2010)

$\mathrm{Cyc}_{2}-\mathrm{Cyc}_{1}-\mathrm{RcY}-\mathrm{aa}_{3} \mathrm{Fe}(\mathrm{II}): \mathrm{O}_{2}$ OR

A. ferroooxidans

(Castelle et al., 2008)

\begin{tabular}{|ccll|}
\hline $\begin{array}{c}\text { Anerobic respiratory } \\
\text { supercomplexes } \\
\text { HYD-SR }\end{array}$ & Activity & Microorganisms & References \\
& $\mathrm{H}_{2}:: \mathrm{S}^{0} \mathrm{OR}$ & $\begin{array}{l}\text { A. aeolicus } \\
\text { P. abyssi } \\
\text { T. neutrophilus } \\
\text { A. ambivalens }\end{array}$ & $\begin{array}{l}\text { (Guiral et al., 2005), } \\
\text { (Dirmeier et al., 1998), } \\
\text { (Laska and Kletzin, 2000) } \\
\text { (Laska et al., 2003) }\end{array}$ \\
& & & \\
& & & \\
& & & \\
& &
\end{tabular}

